\title{
GENERALIZED LYUBEZNIK NUMBERS
}

\author{
LUIS NÚNNEZ-BETANCOURT AND EMILY E. WITT
}

\begin{abstract}
Given a local ring containing a field, we define and investigate a family of invariants that includes the Lyubeznik numbers but captures finer information. These generalized Lyubeznik numbers are defined in terms of $D$-modules and are proved well defined using a generalization of the classical version of Kashiwara's equivalence for smooth varieties; we also give a definition for finitely generated $K$-algebras. These new invariants are indicators of $F$-singularities in characteristic $p>0$ and have close connections with characteristic cycle multiplicities in characteristic zero. We characterize the generalized Lyubeznik numbers associated to monomial ideals and compute examples of those associated to determinantal ideals.
\end{abstract}

\section{$\S 1$. Introduction}

The aim of this article is to define and study a family of invariants of a local ring containing a field. This family includes the Lyubeznik numbers but captures finer information than this smaller family. For example, although the Lyubeznik numbers cannot differentiate between two 1dimensional rings or two complete intersection rings, the new invariants can.

These generalized Lyubeznik numbers are defined in terms of lengths of certain local cohomology modules in a category of $D$-modules. To prove that they are well defined, we use a generalization of the classical version of Kashiwara's equivalence for smooth varieties. As a consequence of this approach, our work also gives a new proof that the Lyubeznik numbers are well defined.

Recall that if $R$ is a local ring with residue field $K$ admitting a surjection from an $n$-dimensional regular local ring $S$ containing a field, and if $I$ is its kernel, the Lyubeznik number of $R$ with respect to $i, j \in \mathbb{N}$ is defined as

$$
\lambda_{i, j}(R):=\operatorname{dim}_{K} \operatorname{Ext}_{S}^{i}\left(K, H_{I}^{n-j}(S)\right) .
$$

Received October 23, 2012. Revised April 30, 2013. Accepted August 19, 2013.

First published online July 23, 2014.

2010 Mathematics Subject Classification. Primary 13D45, 13N10; Secondary 13H99.

(C) 2014 by The Editorial Board of the Nagoya Mathematical Journal 
For $R$ any local ring containing a field, $\lambda_{i, j}(R):=\lambda_{i, j}(\widehat{R})$, where $\widehat{R}$ is the completion of $R$ with respect to its maximal ideal. Remarkably, these invariants do not depend on the choice of $S$ or on the surjection (see [L1, Theorem 4.1]).

The Lyubeznik numbers carry ring-theoretic information. For example, if $d$ denotes the dimension of $R, \lambda_{d, d}(R)$ is nonzero and $\lambda_{i, j}(R)$ vanishes if either $i>d$ or $j>d$ (see [L1, Properties 4.4]). Moreover, if $R$ is CohenMacaulay, then $\lambda_{d, d}(R)=1$ (see [K2, Theorem 1]). The Lyubeznik numbers also have extensive geometric and topological interpretations (see, e.g., [BlB], [GS], [K1], [W1], [Z]).

The importance of the properties that the Lyubeznik numbers detect has motivated generalizations of the invariants. For example, Àlvarez Montaner [A2, Definition 3.6] defined a larger family of invariants of local rings admitting a surjection from a power series ring over a field of characteristic zero; the definition is in terms of characteristic cycle multiplicities (see Definition 2.2) of certain local cohomology modules.

We define a family of invariants of a local ring of any characteristic containing a field. In the definition of the Lyubeznik numbers, if $E_{S}(K)$ denotes the injective hull of $K$ as an $S$-module, then $H_{m}^{i} H_{I}^{n-j}(S) \cong \bigoplus_{i=1}^{\alpha} E_{S}(K)$ for some $\alpha \geq 0$, and $\lambda_{i, j}(R)=\alpha$ (see [L1, Lemma 1.4, Corollary 3.6]). As $E_{S}(K)$ is a simple $D(S, K)$-module, $\lambda_{i, j}(R)=\operatorname{length}_{D(S, K)} H_{m}^{i} H_{I}^{n-j}(S)$, where $D(S, K)$ is the ring of $K$-linear differential operators on $S$ (see Section 2). This motivates the definition of the new invariants, which are finite by [L3, Corollary 6].

Theorem/Definition (see Theorem 4.2, Definition 4.3). Let $(R, m, K)$ be a local ring containing a field, let $\widehat{R}$ be its completion at $m$, and let $K^{\prime}$ be a coefficient field of $\widehat{R}$. Then $\widehat{R}$ admits a surjection $\pi: S \rightarrow \widehat{R}$, where $S=K\left[\left[x_{1}, \ldots, x_{n}\right]\right]$ for some $n \geq 1$, and such that $\pi(K)=K^{\prime}$. For $1 \leq j \leq s$, fix $i_{j} \in \mathbb{N}$ and ideals $I_{j} \subseteq R$, and let $J_{j}=\pi^{-1}\left(I_{j} \widehat{R}\right) \subseteq S$. The generalized Lyubeznik number of $R$ with respect to $K^{\prime}, I_{1}, \ldots, I_{s}$, and $i_{1}, \ldots, i_{s}$,

$$
\lambda_{I_{s}, \ldots, I_{1}}^{i_{s}, \ldots, i_{1}}\left(R ; K^{\prime}\right):=\operatorname{length}_{D(S, K)} H_{J_{s}}^{i_{s}} \cdots H_{J_{2}}^{i_{2}} H_{J_{1}}^{n-i_{1}}(S)
$$

is finite and depends only on $R, K^{\prime}, I_{1}, \ldots, I_{s}$, and $i_{1}, \ldots, i_{s}$.

Although the generalized Lyubeznik numbers a priori depend on the choice of a coefficient field of $\widehat{R}$, sometimes only one such field exists. For example, this happens when $K$ is a perfect field of characteristic $p>0$. 
Whether it is possible in general to avoid such a dependence relies on an open question of Lyubeznik (see Question 4.6; cf. [L4]).

A crucial component in proving that the generalized Lyubeznik numbers are well defined is the following equivalence of categories, which is a generalization of Kashiwara's classical equivalence for smooth varieties (see [Co], [B2], $[\mathrm{H}])$.

TheOrem (see Theorems 3.4 and 3.13). Let $R$ be a Noetherian ring and let $S=R[[x]]$. Let $\mathcal{C}$ denote the category of $R$-modules, and let $\mathcal{D}$ denote the category of $D(S, R)$-modules that are supported on the Zariski-closed subset $\mathcal{V}(x S)$ of $\operatorname{Spec}(S)$. Then the functor

$$
\begin{aligned}
G: \mathcal{C} & \rightarrow \mathcal{D}, \\
M & \mapsto M \otimes_{R} S_{x} / S
\end{aligned}
$$

is an equivalence of categories, with inverse functor $\widetilde{G}: \mathcal{D} \rightarrow \mathcal{C}$ given by $\widetilde{G}(N)=\operatorname{Ann}_{N}(x S)$.

Moreover, in the setting that $K$ is a field and $R=K\left[\left[y_{1}, \ldots, y_{n}\right]\right]$, so that $S=K\left[\left[y_{1}, \ldots, y_{n}, x\right]\right]$, then $G$ is an equivalence from the category of $D(R, K)$-modules to the category of $D(S, K)$-modules supported on $\mathcal{V}(x S)$.

The family of generalized Lyubeznik numbers does, in fact, contain the Lyubeznik numbers, and consequently, we give a new proof that the Lyubeznik numbers are well defined. Vanishing results analogous to those for the Lyubeznik numbers hold for the new invariants, and they behave well under finite field extensions.

Results of Blickle [Bl, Theorem 4.9, Corollaries 4.10, 4.16] enable, in certain cases, straightforward characterizations of $F$-regularity and $F$-rationality in terms of certain generalized Lyubeznik numbers, and recent results of the first author and Pérez $[\mathrm{NP}]$ imply that these invariants provide a measure on how "far" an $F$-pure hypersurface is from being $F$-regular.

We characterize the generalized Lyubeznik numbers associated to monomial ideals as certain lengths in a category of straight modules, and in characteristic zero also in terms of characteristic cycle multiplicities. Examples of the (generalized) Lyubeznik numbers associated to certain determinantal ideals are computed, which illustrate their dependence on characteristic.

We also define a global version of generalized Lyubeznik numbers of finitely generated $K$-algebras; the authors are not aware of such a previously defined global notion. 
Definition (see Definition 4.4). Let $R$ be a finitely generated $K$-algebra. Then $R$ admits a surjection of $K$-algebras $\pi: S \rightarrow R$, where we have that $S=K\left[x_{1}, \ldots, x_{n}\right]$ for some $n \in \mathbb{N}$. For $1 \leq j \leq s$, fix $i_{j} \in \mathbb{N}$ and ideals $I_{j} \subseteq R$, and let $J_{j}=\pi^{-1}\left(I_{j}\right) \subseteq S$. Then the generalized Lyubeznik number of $R$ with respect to $I_{1}, \ldots, I_{s}$ and $i_{1}, \ldots, i_{s}$,

$$
\lambda_{I_{s}, \ldots, I_{1}}^{i_{s}, \ldots, i_{1}}(R):=\operatorname{length}_{D(S, K)} H_{J_{s}}^{i_{s}} \cdots H_{J_{2}}^{i_{2}} H_{J_{1}}^{n-i_{1}}(S),
$$

is finite and depends only on $R, I_{1}, \ldots, I_{s}$, and $i_{1}, \ldots, i_{s}$ (not on $S$ nor on $\pi$ ).

\section{$\S 2 . \quad D$-module preliminaries}

In this section, we recall some relevant facts about $D$-modules. We refer the reader to $[\mathrm{B} 2],[\mathrm{B} 1],[\mathrm{Co}]$, and [MN] for details.

Given rings $A \subseteq S$, we define the ring of $A$-linear differential operators on $S, D(S, A)$, as the subring of $\operatorname{Hom}_{A}(S, S)$ defined inductively as follows. The differential operators of order zero are given by multiplication by elements in $S$. An element $\theta \in \operatorname{Hom}_{A}(S, S)$ is a differential operator of order less than or equal to $k+1$ if, for every $r \in S,[\theta, r]:=\theta \cdot r-r \cdot \theta$ is a differential operator of order less than or equal to $k$. From the definition, we see that if $B$ is a subring of $A$, then $D(S, B) \subseteq D(S, A)$.

If $M$ is a $D(S, A)$-module, then $M_{f}$ has the structure of a $D(S, A)$-module such that, for every $f \in S$, the natural map $M \rightarrow M_{f}$ is a $D(S, A)$-module homomorphism. As a result, since $S$ is a $D(S, A)$-module, for all ideals $I_{1}, \ldots, I_{s} \subseteq S$, and all $i_{1}, \ldots, i_{s} \in \mathbb{N}, H_{I_{s}}^{i_{s}} \cdots H_{I_{2}}^{i_{2}} H_{I_{1}}^{i_{1}}(S)$ is also a $D(S, A)$ module (see [L1, Example 2.1(iv)]).

If $S=A\left[\left[x_{1}, \ldots, x_{n}\right]\right]$, then $D(S, A)=S\left\langle\frac{1}{t !} \frac{\partial^{t}}{\partial x_{i} t} \mid t \in \mathbb{N}, 1 \leq i \leq n\right\rangle \subseteq$ $\operatorname{Hom}_{A}(S, S)$ (see [Gr, Theorem 16.12.1]). Moreover, if $A=K$ is a field, then $S_{f}$ has finite length in the category of $D(S, K)$-modules for every $f \in S$. Consequently, every module of the form $H_{I_{s}}^{i_{s}} \cdots H_{I_{2}}^{i_{2}} H_{I_{1}}^{i_{1}}(S)$ also has finite length in this category (see [L3, Corollary 6]).

Hypothesis 2.1. Throughout the remainder of this section, we will assume that $S$ is either $K\left[x_{1}, \ldots, x_{n}\right]$ or $K\left[\left[x_{1}, \ldots, x_{n}\right]\right]$, where $K$ is a field of characteristic zero. Let $D=D(S, K)$.

In our case, $D=S\left\langle\frac{\partial}{\partial x_{1}}, \ldots, \frac{\partial}{\partial x_{n}}\right\rangle \subseteq \operatorname{Hom}_{K}(S, S)$, and there is an ascending filtration

$$
\Gamma_{i}:=\{\delta \in D \mid \operatorname{ord}(\delta) \leq i\}=\bigoplus_{\alpha_{1}+\cdots+\alpha_{n} \leq i} S \cdot \frac{\partial^{\alpha}}{\partial x_{i}^{\alpha}} .
$$


Moreover, $\operatorname{gr}^{\Gamma}(D) \cong S\left[y_{1}, \ldots, y_{n}\right]$, a polynomial ring over $S$. A filtration $\Omega=\left\{\Omega_{j}\right\}$ of $S$-modules on a $D$-module $M$ is a good filtration if $\Omega_{j} \subseteq \Omega_{j+1}$, $\bigcup_{j \in \mathbb{N}} \Omega_{j}=M, \Gamma_{i} \Omega_{j} \subseteq \Omega_{i+j}$, and $\operatorname{gr}^{\Omega}(M)=\bigoplus_{j \in \mathbb{N}} \Omega_{j+1} / \Omega_{j}$ is a finitely generated $\operatorname{gr}^{\Gamma}(D)$-module.

If $\Omega$ is a good filtration, neither $\operatorname{dim}_{\operatorname{gr}^{\Omega}(D)} \operatorname{gr}^{\Omega}(M) \operatorname{nor} \operatorname{Rad}\left(\operatorname{Ann}_{\operatorname{gr}^{\Omega}(D)} \times\right.$ $\left.\mathrm{gr}^{\Omega}(M)\right)$ depends on the choice of good filtration. For notational brevity, we will omit the filtration when referring to the associated graded ring or module.

A finitely generated $D$-module $M$ is holonomic if either $M=0$ or $\operatorname{dim}_{\operatorname{gr}(D)} \operatorname{gr}(M)=n$. The holonomic $D$-modules form a full abelian subcategory of the category of $D$-modules, and every holonomic $D$-module has finite length as a $D$-module. Moreover, if $M$ is holonomic, then $M_{f}$ is also holonomic for every $f \in S$. As a consequence, since $S$ is holonomic, every module of the form $H_{I_{s}}^{i_{s}} \cdots H_{I_{2}}^{i_{2}} H_{I_{1}}^{i_{1}}(S)$ is also holonomic.

DEFINITION 2.2 (Characteristic variety, characteristic cycle, characteristic cycle multiplicity). Given a holonomic $D$-module $M$, the characteristic variety of $M$ is

$$
C(M)=\mathcal{V}\left(\operatorname{Rad}\left(\operatorname{Ann}_{\operatorname{gr}(D)} \operatorname{gr}(M)\right)\right) \subseteq \operatorname{Spec} \operatorname{gr}(D),
$$

and the characteristic cycle of $M$ is $C C(M)=\sum m_{i} V_{i}$, where the sum is taken over all the irreducible components $V_{i}$ of $C(M)$, and $m_{i}$ is the corresponding multiplicity. Moreover, the (characteristic cycle) multiplicity of $M$ is $e(M)=\sum m_{i}$.

REMARK 2.3. If $0 \rightarrow M^{\prime} \rightarrow M \rightarrow M^{\prime \prime} \rightarrow 0$ is an exact sequence of holonomic $D$-modules, then $C C(M)=C C\left(M^{\prime}\right)+C C\left(M^{\prime \prime}\right)$; as a consequence, $e(M)=e\left(M^{\prime}\right)+e\left(M^{\prime \prime}\right)$. In addition, $C C(M)=0$ if and only if $M=0$, so that $e(M)=0$ if and only if $M=0$ as well.

\section{§3. A Kashiwara equivalence}

In this section, we study a functor utilized by Lyubeznik to prove that his original invariants are well defined (see [L1, Lemma 4.3]). In order to prove that the generalized Lyubeznik numbers are well defined, significant development of the theory of this functor (the "direct image" functor) is necessary. The fact that this functor is, in fact, an equivalence to a certain category of $D$-modules is essential to the results here, as we will see in Theorem 3.4. 
Definition 3.1 (Key functor $G$ ). Let $R$ be a Noetherian ring, and let $S=R[[x]]$. Let $G: R$-mod $\rightarrow S$-mod denote the functor that will be given here by $G(-)=(-) \otimes_{R} S_{x} / S$.

Remark 3.2. For every element $u \in G(M)$ there exist $\ell, \alpha_{1}, \ldots, \alpha_{\ell} \in \mathbb{N}$ and $m_{1}, \ldots, m_{\ell} \in M$, uniquely determined, such that $u=m_{\ell} \otimes x^{-\alpha_{\ell}}+\cdots+$ $m_{1} \otimes x^{-\alpha_{1}}$ and $m_{\ell} \neq 0$, because

$$
G(M)=M \otimes_{R} S_{x} / S=M \otimes_{R}\left(\bigoplus_{\alpha \in \mathbb{N}} R x^{-\alpha}\right)=\bigoplus_{\alpha \in \mathbb{N}}\left(M \otimes R x^{-\alpha}\right)
$$

Moreover, $G$ is an exact functor and commutes with local cohomology.

REMARK 3.3. In fact, $G$ is a functor from the category $R$-modules to the category of $D(S, R)$-modules. Indeed, we will let $M$ be a $D(S, R)$ module. Since $D(S, R)=S\left\langle\frac{1}{t !} \frac{\partial^{t}}{\partial x^{t}} \mid t \in \mathbb{N}\right\rangle \subseteq \operatorname{Hom}_{K}(S, S)$, it is enough to give an action of each $\frac{1}{t !} \frac{\partial^{t}}{\partial x^{t}}$ on $G(M)$. If $m \otimes x^{-\alpha} \in G(M)$, we define

$$
\left(\frac{1}{t !} \frac{\partial^{t}}{\partial x^{t}}\right) \cdot\left(m \otimes x^{-\alpha}\right)=\left(\begin{array}{c}
\alpha+t-1 \\
t
\end{array}\right) \cdot\left((-1)^{t} m \otimes x^{-\alpha-t}\right) .
$$

In particular, taking $\alpha=1$ and $t=\beta$, we see that, for every $\beta \in \mathbb{N}$,

$$
m \otimes x^{-\beta}=\frac{(-1)^{\beta-1}}{(\beta-1) !} \frac{\partial^{\beta-1}}{\partial x^{\beta-1}}\left(m \otimes x^{-1}\right) .
$$

Similarly, for every $R$-module homomorphism $\varphi, G(\varphi)=\varphi \otimes_{R} S_{x} / S$ is a homomorphism of $D(S, R)$-modules.

We prove an equivalence of categories that gives a general treatment of Kashiwara's equivalence theorem. This equivalence is known for polynomial rings or, more generally, for smooth varieties over a field (see [Co], [B2], [H]). However, the authors could not find the statement presented in Theorem 3.4 in the literature and include this result for completeness. We point out that this result does not require that $R$ contain a field.

Theorem 3.4. Let $R$ be a Noetherian ring, and let $S=R[[x]]$. Let $\mathcal{C}$ denote the category of $R$-modules, and let $\mathcal{D}$ denote the category of $D(S, R)$ modules that are supported on the Zariski-closed subset $\mathcal{V}(x S)$ of $\operatorname{Spec}(S)$. Then $G: \mathcal{C} \rightarrow \mathcal{D}$ as in Definition 3.1 is an equivalence of categories with inverse functor $\widetilde{G}: \mathcal{D} \rightarrow \mathcal{C}$ given by $\widetilde{G}(M)=\operatorname{Ann}_{M}(x S)$. 
Proof. It is clear that for every $R$-module $M, \widetilde{G}(G(M))$ is naturally isomorphic to $M$. It suffices to prove that for every $D(S, R)$-module $N$ with support on $\mathcal{V}(x S), G(\widetilde{G}(N))$ is naturally isomorphic to $N$. Let $M=\widetilde{G}(N)=$ $\operatorname{Ann}_{N}(x S)$, and let $\phi: G(M) \rightarrow N$ be the $R$-module homomorphism defined on simple tensors by $m \otimes x^{-\alpha} \mapsto \frac{(-1)^{\alpha-1}}{(\alpha-1) !} \frac{\partial^{\alpha-1}}{\partial x^{\alpha-1}} m$. We will prove, in steps, that $\phi$ is an isomorphism of $D(S, R)$-modules.

First, we will show that $\phi$ is a $D(S, R)$-module homomorphism. Since $D(S, R)=S\left\langle\frac{1}{t !} \frac{\partial^{t}}{\partial x^{t}} \mid t \in \mathbb{N}\right\rangle$, it is enough to show that $\phi$ commutes with multiplication by $x$ and by any $\frac{1}{t !} \frac{\partial^{t}}{\partial x^{t}}$.

We first prove commutativity with $\frac{1}{t !} \frac{\partial^{t}}{\partial x^{t}}$. Now, for any $t \in \mathbb{N}$,

$$
\begin{aligned}
\phi\left(\frac{1}{t !} \frac{\partial^{t}}{\partial x^{t}}\left(m \otimes x^{-\alpha}\right)\right) & =\phi\left(\left(\begin{array}{c}
\alpha+t-1 \\
t
\end{array}\right)\left((-1)^{t} m \otimes x^{-\alpha-t}\right)\right) \\
& =\left(\begin{array}{c}
\alpha+t-1 \\
t
\end{array}\right) \frac{(-1)^{\alpha-1}}{(\alpha+t-1) !} \frac{\partial^{\alpha+t-1}}{\partial x^{\alpha+t-1}} m \\
& =\frac{1}{t !} \frac{(-1)^{\alpha-1}}{(\alpha-1) !} \frac{\partial^{\alpha+t-1}}{\partial x^{\alpha+t-1}} m \\
& =\frac{1}{t !} \frac{\partial^{t}}{\partial x^{t}}\left(\frac{(-1)^{\alpha-1}}{(\alpha-1) !} \frac{\partial^{\alpha-1}}{\partial x^{\alpha-1}} m\right) \\
& =\frac{1}{t !} \frac{\partial^{t}}{\partial x^{t}} \phi\left(m \otimes x^{-\alpha}\right) .
\end{aligned}
$$

To prove that the map commutes with $x$, note that for every $t \in \mathbb{N}$,

$$
x \frac{1}{t !} \frac{\partial^{t}}{\partial x^{t}}-\frac{1}{t !} \frac{\partial^{t}}{\partial x^{t}} x=-\frac{1}{(t-1) !} \frac{\partial^{t-1}}{\partial x^{t-1}}
$$

as differential operators. We conclude that

$$
\begin{aligned}
\phi & \left(x\left(m \otimes x^{-\alpha}\right)\right) \\
& =\phi\left(m \otimes x^{-\alpha+1}\right)=\phi\left(m \otimes \frac{(-1)^{\alpha-2}}{(\alpha-2) !} \frac{\partial^{\alpha-2}}{\partial x^{\alpha-2}} x^{-1}\right) \\
(3.4 .1) \quad & =\frac{(-1)^{\alpha-2}}{(\alpha-2) !} \frac{\partial^{\alpha-2}}{\partial x^{\alpha-2}} \phi\left(m \otimes x^{-1}\right) \\
& =x \frac{(-1)^{\alpha-1}}{(\alpha-1) !} \frac{\partial^{\alpha-1}}{\partial x^{\alpha-1}} \phi\left(m \otimes x^{-1}\right)-\frac{(-1)^{\alpha-1}}{(\alpha-1) !} \frac{\partial^{\alpha-1}}{\partial x^{\alpha-1}} x \phi\left(m \otimes x^{-1}\right)
\end{aligned}
$$




$$
\begin{aligned}
& =x \frac{(-1)^{\alpha-1}}{(\alpha-1) !} \frac{\partial^{\alpha-1}}{\partial x^{\alpha-1}} \phi\left(m \otimes x^{-1}\right) \\
& =x \phi\left(m \otimes \frac{(-1)^{\alpha-1}}{(\alpha-1) !} \frac{\partial^{\alpha-1}}{\partial x^{\alpha-1}} x^{-1}\right) \\
& =x \phi\left(m \otimes x^{-\alpha}\right)
\end{aligned}
$$

where (3.4.1) and (3.4.2) are due to the commutativity with $\frac{1}{t !} \frac{\partial^{t}}{\partial x^{t}}$.

It remains to prove that $\phi$ is bijective; we proceed by contradiction. Suppose that there exists $u=m_{\ell} \otimes x^{-\alpha_{\ell}}+\cdots+m_{1} \otimes x^{-\alpha_{1}} \in \operatorname{Ker}(\phi)$ such that $m_{\ell} \neq 0$. Then $\phi\left(m_{\ell} \otimes x^{-1}\right)=\phi\left(x^{\ell-1} u\right)=x^{\ell-1} \phi(u)=0$. Thus, $m_{\ell}=0$ because $\left.\phi\right|_{M \otimes R x^{-1}}$ is bijective, which is a contradiction.

We now see that $\phi\left(\operatorname{Ann}_{G(M)}\left(x^{j} S\right)\right)=\operatorname{Ann}_{N}\left(x^{j} S\right)$ for every $j \geq 1$ by induction, which will imply that $\phi$ is surjective (since $N$ is supported on $\mathcal{V}(x S))$. Since $\phi\left(\operatorname{Ann}_{G(M)}\left(x^{j} S\right)\right) \subseteq \operatorname{Ann}_{N}\left(x^{j} S\right)$ for all $j$, we seek the opposite inclusion. For $j=1$, take $n \in M=\operatorname{Ann}_{N}(x S)$; then $n \otimes x^{-1} \in$ $G(M)$, so $\phi\left(n \otimes x^{-1}\right)=n$. Now take any $j \geq 1$ and assume that the statement holds for $j-1$. For any $u \in \operatorname{Ann}_{N}\left(x^{j} S\right), x u \in \operatorname{Ann}_{N}\left(x^{j-1} S\right)$, so $x u=\phi(v)$ for some $v=m_{j-1} \otimes x^{-j+1}+\cdots+m_{1} \otimes x^{-1} \in G(M)$ by the inductive hypothesis. Let $w=m_{j-1} x^{-j}+\cdots+m_{1} \otimes x^{-2}$. Thus, $x \phi(w)=\phi(x w)=$ $\phi(v)=x u$. This means that $x(\phi(w)-u)=0$, and so $\phi(w)-u \in \operatorname{Ann}_{N}(x S)=$ $\phi\left(\operatorname{Ann}_{G(M)}(x S)\right)$ and $\phi\left(m^{\prime} \otimes x^{-1}\right)=\phi(w)-u$ for some $m^{\prime} \in M$ by the base case. Therefore, $u=\phi\left(w-m \otimes x^{-1}\right) \in \phi\left(\operatorname{Ann}_{G(M)}\left(x^{j} S\right)\right)$.

Proposition 3.5. Let $R$ be a Noetherian ring, and let $S=R[[x]]$. Then $M$ is a finitely generated $R$-module if and only if $G(M)$ is a finitely generated $D(S, R)$-module.

Proof. Given $m_{1}, \ldots, m_{s} \in M$, generators for $M$ as an $R$-module, $m_{1} \otimes$ $x^{-1}, \ldots, m_{s} \otimes x^{-1}$ generate $G(M)$ as a $D(S, R)$-module; by (3.3.1), for $\beta \in \mathbb{N}$, $m_{i} \otimes x^{-\beta}=\frac{(-1)^{\beta-1}}{(\beta-1) !} \frac{\partial^{\beta-1}}{\partial x^{\beta-1}}\left(m_{i} \otimes x^{-1}\right)$, and the set $\left\{m_{i} \otimes x^{-\beta} \mid 1 \leq i \leq s, \beta \in \mathbb{N}\right\}$ generates $G(M)$ as an $R$-module.

If $u_{1}, \ldots, u_{s} \in G(M)$ generate $G(M)$ as a $D(S, R)$-module, then each $u_{i}$ can be written as $u_{i}=m_{i, 1} \otimes x^{-1}+m_{i, 2} \otimes x^{-2}+\cdots+m_{i, \ell_{i}} \otimes x^{-\ell_{i}}$ for some $\ell_{i} \in \mathbb{N}$ and $m_{i, j} \in M$. Then $\left\{m_{i, j} \otimes x^{-j} \mid 1 \leq i \leq s, 1 \leq j \leq \ell_{i}\right\}$ is also a set of generators for $G(M)$ as a $D(S, R)$-module. Since $m_{i, j} \otimes x^{-j}=$ $\frac{(-1)^{j-1}}{(j-1) !} \frac{\partial^{j-1}}{\partial x^{j-1}}\left(m_{i, j} \otimes x^{-1}\right)$, the decomposition in (3.2.1) implies that the $m_{i, j}$ must generate $M$. 
Corollary 3.6. Let $R$ be a Noetherian ring, let $M$ be an $R$-module, and let $S=R[[x]]$. Then length $R(M)=\operatorname{length}_{D(S, R)} G(M)$.

Proof. If $M$ is a simple nonzero $R$-module, then $G(M)$ is a simple $D(S, R)$-module since the $D(S, R)$-submodules of $G(M)$ correspond precisely to $R$-submodules of $M$ by Theorem 3.4. Now say that $\operatorname{length}_{R}(M)=$ $h<\infty$, so that we have a filtration of $R$-modules $0=M_{0} \subsetneq M_{1} \subsetneq \cdots \subsetneq$ $M_{h}=M$ such that each $M_{j+1} / M_{j}$ is a simple $R$-module. Then $0=G\left(M_{0}\right) \subseteq$ $G\left(M_{1}\right) \subseteq \cdots \subseteq G\left(M_{h}\right)=G(M)$ is a filtration of $D(S, R)$-modules such that $G\left(M_{j+1}\right) / G\left(M_{j}\right) \cong G\left(M_{j+1} / M_{j}\right)$ is a simple $D(S, R)$-module for every $j$ by our initial argument. Therefore, $\operatorname{length}_{D(S, R)} G(M)=h$. Similarly, if length $_{R}(M)=\infty$, then length $D(S, R) G(M)=\infty$.

REMARK 3.7. In the following work, we often make use of the following observation: for $R$ a ring and $S=R[[x]]$, if $P$ is a prime ideal of $R$, then $(P, x) S$ is a prime ideal of $S$ since $S /(P, x) S=R / P$ is a domain.

Proposition 3.8. Let $R$ be a Noetherian ring, let $M$ be an $R$-module, and let $S=R[[x]]$. Then $\operatorname{Ass}_{S} G(M)=\left\{(P, x) S \mid P \in \operatorname{Ass}_{R} M\right\}$.

Proof. Let $Q \in \operatorname{Ass}_{S} G(M)$, so that $Q=\operatorname{Ann}_{S} u$ for some $u \in G(M)$. As $H_{x S}^{0}(G(M))=G(M), x \in Q$. Thus, $u \in \operatorname{Ann}_{G(M)} x S \cong M$. (The isomorphism is due to Theorem 3.4.) Moreover, we have the natural epimorphism $R \rightarrow S / Q$ with kernel $P=\operatorname{Ann}_{R} u \in \operatorname{Ass}_{R} M$. Thus, $Q=(P, x) S$.

Take $Q=(P, x) S$, where $P=\operatorname{Ann}_{R} u \in \operatorname{Ass}_{R} M, u \in M$. Then $Q=$ $\operatorname{Ann}_{S}\left(u \otimes x^{-1}\right)$. Hence, $Q \in \operatorname{Ass}_{S} G(M)$.

Lemma 3.9. Let $R$ be a Noetherian ring, let $M$ be an $R$-module, and let $S=R[[x]]$. Then for every ideal $I \subseteq R$ and all $j \in \mathbb{N}, G\left(H_{I}^{j}(M)\right) \cong$ $H_{(I, x) S}^{j+1}\left(M \otimes_{R} S\right)$.

Proof. Since $S$ and $S_{x}$ are flat $R$-algebras and $S_{x} / S$ is a free $R$-module, we know that $H_{I}^{j}(M) \otimes_{R} S \cong H_{I S}^{j}\left(M \otimes_{R} S\right), H_{I}^{j}(M) \otimes_{R} S_{x} \cong H_{I S}^{j}\left(M \otimes_{R} S_{x}\right)$, and $H_{I}^{j}(M) \otimes_{R} S_{x} / S \cong H_{I S}^{j}\left(M \otimes_{R} S_{x} / S\right)$. Moreover, the sequence

$$
0 \rightarrow H_{I S}^{j}\left(M \otimes_{R} S\right) \rightarrow H_{I S}^{j}\left(M \otimes_{R} S_{x}\right) \rightarrow H_{I S}^{j}\left(M \otimes_{R} S_{x} / S\right) \rightarrow 0
$$

is exact, so $G\left(H_{I}^{j}(M)\right) \cong H_{I S}^{j}\left(M \otimes_{R} S_{x}\right) / H_{I S}^{j}\left(M \otimes_{R} S\right)$.

On the other hand, we have a long exact sequence

$$
\cdots \rightarrow H_{(I, x) S}^{j}\left(M \otimes_{R} S\right) \rightarrow H_{I S}^{j}\left(M \otimes_{R} S\right) \rightarrow H_{I S}^{j}\left(M \otimes_{R} S_{x}\right) \rightarrow \cdots
$$


Since $H_{I S}^{j}\left(M \otimes_{R} S\right) \rightarrow H_{I S}^{j}\left(M \otimes_{R} S_{x}\right)$ is injective by (3.9.1), the long sequence splits into short exact sequences

$$
0 \rightarrow H_{I S}^{j}\left(M \otimes_{R} S\right) \rightarrow H_{I S}^{j}\left(M \otimes_{R} S_{x}\right) \rightarrow H_{(I, x) S}^{j+1}\left(M \otimes_{R} S\right) \rightarrow 0 .
$$

Hence, $G\left(H_{I}^{j}(M)\right) \cong H_{(I, x) S}^{j+1}\left(M \otimes_{R} S\right)$.

Proposition 3.10. Let $(R, m, K)$ be a Noetherian local ring, let $M$ be an $R$-module, and let $S=R[[x]]$. Fix $I_{1}, \ldots, I_{s}$ ideals of $R$ and $j_{1}, \ldots j_{s} \in \mathbb{N}$. Then

$$
G\left(H_{I_{s}}^{j_{s}} \cdots H_{I_{2}}^{j_{2}} H_{I_{1}}^{j_{1}}(M)\right) \cong H_{\left(I_{s}, x\right) S}^{j_{s}} \cdots H_{\left(I_{2}, x\right) S}^{j_{2}} H_{\left(I_{1}, x\right) S}^{j_{1}+1}\left(M \otimes_{R} S\right) .
$$

Proof. We proceed by induction on $s$. If $s=1$, the statement follows from Lemma 3.9. Suppose that it holds for some $s \geq 1$. Let $N_{\ell}=H_{I_{\ell}}^{j_{\ell}} \ldots$ $H_{I_{2}}^{j_{2}} H_{I_{1}}^{j_{1}}(M)$ for $1 \leq \ell \leq s+1$, so we need to prove that $G\left(N_{s+1}\right) \cong$ $H_{\left(I_{s+1}, x\right) S}^{j_{s+1}}\left(G\left(N_{s}\right)\right)$. Now,

$$
G\left(N_{s+1}\right)=H_{I_{s+1}}^{j_{s+1}}\left(N_{s}\right) \otimes_{R} S_{x} / S \cong H_{I_{s+1} S}^{j_{s+1}}\left(N_{s} \otimes_{R} S_{x} / S\right)=H_{I_{s+1} S}^{j_{s+1}}\left(G\left(N_{s}\right)\right)
$$

Consider the long exact sequence of functors

$$
\cdots \rightarrow H_{I_{s+1} S}^{j_{s+1}}(-) \rightarrow H_{\left(I_{s+1}, x\right) S}^{j_{s+1}}(-) \rightarrow H_{I_{s+1} S}^{j_{s+1}}\left(-\otimes_{S} S_{x}\right) \rightarrow \cdots
$$

Since $G\left(N_{s}\right)$ is supported on $\mathcal{V}(x S), H_{I_{s+1} S}^{i}\left(G\left(N_{s}\right) \otimes_{S} S_{x}\right)=0$ for all $i \in$ $\mathbb{N}$, and $G\left(N_{s}\right) \otimes_{S} S_{x}=0$. Moreover, $H_{I_{s+1} S}^{j_{s+1}}\left(G\left(N_{s}\right)\right) \cong H_{\left(I_{s+1}, x\right) S}^{j_{s+1}}\left(G\left(N_{s}\right)\right)$. Hence, $G\left(N_{s+1}\right) \cong H_{\left(I_{s+1}, x\right) S}^{j_{s+1}}\left(G\left(N_{s}\right)\right)$.

As $G$ is an equivalence of categories, $G\left(\operatorname{Hom}_{R}(M, N)\right)=$ $\operatorname{Hom}_{D(S, R)}(G(N), G(M))$. Thus, $M$ is an injective $R$-module if and only if $G(M)$ is an injective object in $\mathcal{D}$, the category of $D(S, R)$-modules supported at $\mathcal{V}(x S)$. We now characterize precisely when $G(M)$ is injective as an $S$-module.

Proposition 3.11. Let $S=R[[x]]$, where $R$ is a Gorenstein ring. Given a prime ideal $P$ of $R$, let $E_{R}(R / P)$ denote the injective hull of $R / P$ over $R$. Then $G\left(E_{R}(R / P)\right) \cong E_{S}(S /(P, x) S)$. Moreover, $M$ is an injective $R$-module if and only if $G(M)$ is an injective $S$-module. 
Proof. Let $d=\operatorname{dim}\left(R_{P}\right)$. Since $R$ is a Gorenstein ring, $S_{x} / S$ a flat $R$-module, and $G\left(H_{P}^{d}(R)\right) \cong H_{(P, x) S}^{d+1}(S)$ by Lemma 3.9, we have that

$$
\begin{aligned}
G\left(E_{R}(R / P)\right) & \cong G\left(H_{P R_{P}}^{d}\left(R_{P}\right)\right) \cong G\left(H_{P}^{d}(R) \otimes_{R} R_{P}\right) \\
& \cong G\left(H_{P}^{d}(R)\right) \otimes_{R} R_{P} \cong H_{(P, x) S}^{d+1}\left(S_{P}\right) .
\end{aligned}
$$

As $S_{P} /(P, x) S_{P} \cong R_{P} / P R_{P},(P, x) S_{P}$ is a maximal ideal of the Gorenstein ring $S_{P}$, and

$$
H_{(P, x) S}^{d+1}\left(S_{P}\right) \cong E_{S_{P}}\left(S_{P} /(P, x) S_{P}\right) \cong E_{S}(S /(P, x) S)
$$

that is, $G\left(E_{R}(R / P)\right) \cong E_{S}(S /(P, x) S)$. Moreover, $G$ sends injective $R$-modules to injective $S$-modules because every injective $R$-module is a direct sum of injective hulls of prime ideals.

It remains to prove that if $G(M)$ is an injective $S$-module, then $M$ is an injective $R$-module. This follows because $M=\operatorname{Ann}_{G(M)}(x S)$ by Theorem 3.4; any injection of $R$-modules $\iota: N \hookrightarrow N^{\prime}$ is also an injection of $S$-modules, where $x$ acts by zero. Then any $S$-module map $f: N \rightarrow G(M)$ is an $R$-module map and must have image in $\operatorname{Ann}_{G(M)}(x S)=M$, so the induced map $g: N \rightarrow M$ is a map of $R$-modules such that $f=g \circ \iota$.

Proposition 3.12. Let $R$ be a Gorenstein ring, take $R$-modules $M$ and $N$, and let $S=R[[x]]$. As $R=S / x S, M$ is naturally an $S$-module via extension of scalars, so that for all $i \in \mathbb{N}$, $\operatorname{Ext}_{S}^{i}(M, G(N))$ is an $S$-module annihilated by $x S$, so that it is also naturally an $R$-module. As $R$-modules,

$$
\operatorname{Ext}_{S}^{i}(M, G(N))=\operatorname{Ext}_{R}^{i}(M, N) .
$$

Proof. Let $E^{*}=E^{0} \rightarrow E^{1} \rightarrow \cdots \rightarrow E^{i} \rightarrow \cdots$ be an injective $R$-resolution of $N$. Then $G\left(E^{*}\right)$ is an injective $S$-resolution for $G(N)$ by Proposition 3.11. We notice that $\operatorname{Hom}_{S}(M,-)=\operatorname{Hom}_{S}\left(M, \operatorname{Hom}_{S}(R,-)\right)$ as functors on the category of $S$-modules. Then since $G\left(E_{R}(R / P)\right) \cong E_{S}(S /(P, x) S)$ by Proposition 3.11,

$$
\begin{aligned}
\operatorname{Hom}_{S}\left(M, G\left(E^{*}\right)\right) & =\operatorname{Hom}_{S}\left(M, \operatorname{Hom}_{S}\left(R, G\left(E^{*}\right)\right)\right) \\
& =\operatorname{Hom}_{S}\left(M, E^{*}\right)=\operatorname{Hom}_{R}\left(M, E^{*}\right),
\end{aligned}
$$

and the result follows.

TheOREM 3.13 (Kashiwara's equivalence for power series rings). Let $K$ be a field, let $R=K\left[\left[x_{1}, \ldots, x_{n}\right]\right]$, and let $S=R\left[\left[x_{n+1}\right]\right]$. Let $\mathcal{C}$ denote the 
category of $D(R, K)$-modules, and let $\mathcal{D}$ denote the category of $D(S, K)$ modules that are supported on $\mathcal{V}(x S)$. Then

(i) $\quad G: \mathcal{C} \rightarrow \mathcal{D}$ given by $G(M)=M \otimes_{R} S_{x_{n+1}} / S$ is an equivalence of categories with inverse $\widetilde{G}: \mathcal{D} \rightarrow \mathcal{C}$, where $\widetilde{G}(N)=\operatorname{Ann}_{N}(x S)$;

(ii) $M$ is a finitely generated $D(R, K)$-module if and only if $G(M)$ is a finitely generated $D(S, K)$-module; and

(iii) length $_{D(R, K)} M=$ length $_{D(S, K)} G(M)$.

Proof. The proofs of the statements are analogous to the those of Theorem 3.4, Proposition 3.5, and Corollary 3.6, respectively.

\section{§4. Definitions and first properties}

Remark 4.1. Given a local ring $(R, m, K)$, we say that a field $K^{\prime}$ is a coefficient field of $R$ if $K^{\prime}$ is contained in $R$, and the composition $K^{\prime} \hookrightarrow$ $R \rightarrow R / m=K$ is an isomorphism of fields. Every complete local ring containing a field has a coefficient field by the Cohen structure theorems (see [C, Theorem 9]).

THEOREM 4.2. Let $(R, m, K)$ be a local ring containing a field, and let $\widehat{R}$ be its completion at $m$. Let $K^{\prime}$ be a coefficient field of $\widehat{R}$. Then $\widehat{R}$ admits a surjection $\pi: S \rightarrow \widehat{R}$, where $S=K\left[\left[x_{1}, \ldots, x_{n}\right]\right]$ for some $n \in \mathbb{N}$, and $\pi(K)=K^{\prime}$. For $1 \leq j \leq s$, fix $i_{j} \in \mathbb{N}$ and ideals $I_{j} \subseteq R$, and let $J_{j}=$ $\pi^{-1}\left(I_{j} \widehat{R}\right) \subseteq S$. Then

$$
\text { length }_{D(S, K)} H_{J_{s}}^{i_{s}} \cdots H_{J_{2}}^{i_{2}} H_{J_{1}}^{n-i_{1}}(S)
$$

is finite and depends only on $R, K^{\prime}, I_{1}, \ldots, I_{s}$, and $i_{1}, \ldots, i_{s}$ (not on $S$ nor on $\pi$ ).

Proof. We may assume without loss of generality that $R$ is complete. We know that length ${ }_{D(S, K)} H_{J_{s}}^{j_{s}} \cdots H_{J_{2}}^{j_{2}} H_{J_{1}}^{n-j_{1}}(S)$ is finite by [L3, Corollary 6]. Let $\pi^{\prime}: S^{\prime} \rightarrow R$ be another surjection, where $S^{\prime}=K\left[\left[y_{1}, \ldots, y_{n^{\prime}}\right]\right]$ for some $n^{\prime} \in \mathbb{N}$, and $\pi^{\prime}(K)=K^{\prime}$. Let $J_{1}^{\prime}, \ldots, J_{s}^{\prime}$ be the corresponding preimages of $I_{1}, \ldots, I_{s}$ in $S^{\prime}$ under $\pi^{\prime}$.

Let $S^{\prime \prime}=K\left[\left[z_{1}, \ldots, z_{n+n^{\prime}}\right]\right]$, and let $\pi^{\prime \prime}: S^{\prime \prime} \rightarrow R$ be the surjection defined by

$$
\pi^{\prime \prime}\left(z_{j}\right)= \begin{cases}\pi^{\prime}\left(x_{j}\right) & \text { for } 0 \leq j \leq n \\ \pi^{\prime}\left(y_{j-n}\right) & \text { for } n+1 \leq j \leq n+n^{\prime}\end{cases}
$$

Let $J_{1}^{\prime \prime}, \ldots, J_{s}^{\prime \prime}$ be the corresponding preimages of $I_{1}, \ldots, I_{s}$ in $S^{\prime \prime}$ under $\pi^{\prime \prime}$. 
Let $\alpha: S \rightarrow S^{\prime \prime}$ be the injection given by $\alpha\left(x_{j}\right)=z_{j}$, and note that $\pi^{\prime \prime} \circ \alpha=\pi$. Fix $f_{j} \in S$ such that $\pi\left(f_{j}\right)=\pi^{\prime \prime}\left(z_{n+j}\right)$, for $1 \leq j \leq n^{\prime}$. Then $z_{n+j}-\alpha\left(f_{j}\right) \in \operatorname{Ker}\left(\pi^{\prime \prime}\right)$. The map $\beta: S^{\prime \prime} \rightarrow S$, defined by $\beta\left(z_{j}\right)=x_{j}$ for $1 \leq j \leq n$ and $\beta\left(z_{n+j}\right)=f_{j}$ for $1 \leq j \leq n^{\prime}$, is a splitting of $\alpha$. Then each $z_{n+j}-\alpha\left(f_{j}\right)$ is in $\operatorname{Ker}(\beta)$, and the map

$$
S^{\prime \prime} /\left(z_{n+1}-\alpha\left(f_{1}\right), \ldots, z_{n+n^{\prime}}-\alpha\left(f_{n^{\prime}}\right)\right) \rightarrow S
$$

induced by $\beta$ is an isomorphism, so that $\operatorname{Ker}(\beta)=\left(z_{n+1}-\alpha\left(f_{1}\right), \ldots, z_{n+n^{\prime}}-\right.$ $\left.\alpha\left(f_{n^{\prime}}\right)\right)$. Then $J_{i}^{\prime \prime}=\left(\alpha\left(J_{i}\right)+\left(z_{n+1}-\alpha\left(f_{1}\right), \ldots, z_{n^{\prime}+n}-\alpha\left(f_{n^{\prime}}\right)\right) S^{\prime \prime}\right.$. Since

$$
z_{1}, \ldots, z_{n}, z_{n+1}-\alpha\left(f_{1}\right), \ldots, z_{n^{\prime}+n}-\alpha\left(f_{n^{\prime}}\right)
$$

form a regular system of parameters, we obtain that

$$
\begin{aligned}
& \operatorname{length}_{D\left(S^{\prime \prime}, K\right)} H_{J_{s}^{\prime \prime}}^{i_{s}} \cdots H_{J_{2}^{\prime \prime}}^{i_{2}} H_{J_{1}^{\prime \prime}}^{n^{\prime}+n-i_{1}}\left(S^{\prime \prime}\right) \\
& \quad=\operatorname{length}_{D(S, K)} H_{J_{s}}^{i_{s}} \cdots H_{J_{2}}^{i_{2}} H_{J_{1}}^{n-i_{1}}(S)
\end{aligned}
$$

by Proposition 3.10 and Theorem 3.13. Similarly, length $\operatorname{li}_{D, K)} H_{J_{s}^{\prime}}^{i_{s}} \ldots$ $H_{J_{2}^{\prime}}^{i_{2}} H_{J_{1}^{\prime}}^{n^{\prime}-i_{1}}\left(S^{\prime}\right)$ also equals the left-hand side of $(4.2 .1)$, and the result follows.

Definition 4.3 (Generalized Lyubeznik numbers). Let $(R, m, K)$ be a local ring containing a field, and let $\widehat{R}$ be its completion at $m$. Let $K^{\prime}$ be a coefficient field of $\widehat{R}$. Then $\widehat{R}$ admits a surjection $\pi: S \rightarrow \widehat{R}$, where $S=$ $K\left[\left[x_{1}, \ldots, x_{n}\right]\right]$ for some $n \in \mathbb{N}$, and $\pi(K)=K^{\prime}$. For $1 \leq j \leq s$, fix $i_{j} \in \mathbb{N}$ and ideals $I_{j} \subseteq R$, and let $J_{j}=\pi^{-1}\left(I_{j} \widehat{R}\right) \subseteq S$. Then the generalized Lyubeznik number of $R$ with respect to $K^{\prime}, I_{1}, \ldots, I_{s}$, and $i_{1}, \ldots, i_{s}$,

$$
\lambda_{I_{s}, \ldots, I_{1}}^{i_{s}, \ldots, i_{1}}\left(R ; K^{\prime}\right):=\operatorname{length}_{D(S, K)} H_{J_{s}}^{i_{s}} \cdots H_{J_{2}}^{i_{2}} H_{J_{1}}^{n-i_{1}}(S)
$$

is finite and depends only on $R, K^{\prime}, I_{1}, \ldots, I_{s}$, and $i_{1}, \ldots, i_{s}$ (by Theorem 4.2).

As finitely generated $K$-algebras are endowed with a natural choice of coefficient field, a proof similar to that of Theorem 4.2, using the classical version of Kashiwara's equivalence, admits a global definition of generalized Lyubeznik numbers in this case. In the proof, instead of concluding that the elements $z_{1}, \ldots, z_{n}, z_{n+1}-\alpha\left(f_{1}\right), \ldots, z_{n^{\prime}+n}-\alpha\left(f_{n^{\prime}}\right)$ form a regular system of parameters, we have that $K\left[z_{1}, \ldots, z_{n}, z_{n+1}-\alpha\left(f_{1}\right), \ldots, z_{n^{\prime}+n}-\alpha\left(f_{n^{\prime}}\right)\right]=$ $K\left[z_{1}, \ldots, z_{n^{\prime}+n}\right]$. 
Definition 4.4 (Generalized Lyubeznik numbers of finitely generated $K$-algebras). Let $R$ be a finitely generated $K$-algebra. Then $R$ admits a surjection of $K$-algebras $\pi: S \rightarrow R$, where $S=K\left[x_{1}, \ldots, x_{n}\right]$ for some $n \in \mathbb{N}$. For $1 \leq j \leq s$, fix $i_{j} \in \mathbb{N}$ and ideals $I_{j} \subseteq R$, and let $J_{j}=\pi^{-1}\left(I_{i}\right) \subseteq S$. Then the generalized Lyubeznik number of $R$ with respect to $I_{1}, \ldots, I_{s}$ and $i_{1}, \ldots, i_{s}$,

$$
\lambda_{I_{s}, \ldots, I_{1}}^{i_{s}, \ldots, i_{1}}(R):=\operatorname{length}_{D(S, K)} H_{J_{s}}^{i_{s}} \cdots H_{J_{2}}^{i_{2}} H_{J_{1}}^{n-i_{1}}(S),
$$

is finite and depends only on $R, I_{1}, \ldots, I_{s}$, and $i_{1}, \ldots, i_{s}$, but neither on $S$ nor on $\pi$.

Definition 4.4 gives a global notion of the Lyubeznik numbers; the authors are not aware of a previously defined version in the literature. The treatment of the generalized Lyubeznik numbers for local rings and finitely generated algebras is very similar, but the local case requires a choice of coefficient field. In this paper, we focus on the local case.

REMARK 4.5. In Definition 4.3 (and Theorem 4.2), we rely on a choice of coefficient field $K^{\prime} \subseteq \widehat{R}$. When the election of coefficient field is clear, or when there is only one such field, we often write $\lambda_{I_{s}, \ldots, I_{1}}^{i_{s}, \ldots, i_{1}}(R)$ for $\lambda_{I_{s}, \ldots, I_{1}}^{i_{s}, \ldots, i_{1}}(R$; $\left.K^{\prime}\right)$. In particular, when $(R, m, K)$ is a local ring and $K$ is a perfect field of characteristic $p>0$, if $R^{p^{e}}=\left\{r^{p^{e}} \mid r \in R\right\}$, then $\bigcap_{e \geq 0} R^{p^{e}} \subseteq R$ is the unique coefficient field of $R$.

In general, to decide whether it is possible to avoid the generalized Lyubeznik numbers' dependence on the choice of coefficient field of $\widehat{R}$, we would need to answer the following question, asked by Lyubeznik.

Question 4.6 ([L4, p. 131]). Let $S$ be a complete regular local ring of equal characteristic. For $1 \leq j \leq s$, fix $i_{j} \in \mathbb{N}$ and ideals $J_{j} \subseteq S$. Given any two coefficient fields of $S, K$ and $L$, is

$$
\text { length }_{D(S, K)} H_{J_{s}}^{i_{s}} \cdots H_{J_{2}}^{i_{2}} H_{J_{1}}^{n-i_{1}}(S)=\operatorname{length}_{D(S, L)} H_{J_{s}}^{i_{s}} \cdots H_{J_{2}}^{i_{2}} H_{J_{1}}^{n-i_{1}}(S) \text { ? }
$$

To the best of our knowledge, the answer to Question 4.6 is currently unknown, even in the case when $s=1$.

REMARK 4.7. In Definition 4.3, we may assume that $I_{1} \subseteq \cdots \subseteq I_{s}$, because if an $R$-module $M$ is such that $H_{I}^{0}(M)=M$ for some ideal $I$ of $R$, then $H_{J}^{i}(M)=H_{I+J}^{i}(M)$ for every ideal $J$ of $S$. In addition, $\lambda_{I_{s}, \ldots, I_{1}}^{i_{s}, \ldots, i_{1}}\left(R, K^{\prime}\right)=$ $\lambda_{I_{s}, \ldots, I_{2}, 0}^{i_{s}, \ldots, i_{2}, i_{1}}\left(R / I_{1}, K^{\prime}\right)$.

Proposition 4.8. If $(R, m, K)$ is a local ring containing a field, then $\lambda_{i, j}(R)=\lambda_{m, 0}^{i, j}\left(R ; K^{\prime}\right)$ for any coefficient field $K^{\prime}$ of $\widehat{R}$. 
Proof. Since completion is flat and the Bass numbers are not affected by completion, we may assume that $R$ is complete. Take $S=K\left[\left[x_{1}, \ldots, x_{n}\right]\right]$ such that there exists a surjective ring map $\pi: S \rightarrow R$, such that $\pi(K)=K^{\prime}$. Set $I=\operatorname{Ker}(\pi)=\pi^{-1}(0)$, and notice that the maximal ideal, $\eta$, of $S$ is $\pi^{-1}(m)$. By [L1, Lemma 1.4],

$$
\lambda_{i, j}(R)=\operatorname{dim}_{K} \operatorname{Ext}_{S}^{i}\left(K, H_{I}^{n-j}(S)\right)=\operatorname{dim}_{K} \operatorname{Hom}_{S}\left(K, H_{\eta}^{i} H_{I}^{n-j}(S)\right) .
$$

Since $H_{\eta}^{i} H_{I}^{n-j}(S)$ is isomorphic to a finite direct sum of copies of $E_{S}(K)$ by [L1, Corollary 3.6], and since $E_{S}(K)$ is a simple $D(S, K)$-module (see [L2]), we obtain

$$
\operatorname{dim}_{K} \operatorname{Hom}_{S}\left(K, H_{\eta}^{i} H_{I}^{n-j}(S)\right)=\operatorname{length}_{D(S, K)} H_{\eta}^{i} H_{I}^{n-j}(S)=\lambda_{m, 0}^{i, j}\left(R ; K^{\prime}\right),
$$

and we are done.

Proposition 4.9. Given ideals $I_{1} \subseteq \cdots \subseteq I_{s}$ of a local ring $(R, m, K)$ containing a field, $i_{j} \in \mathbb{N}$ for $1 \leq j \leq s$, and a coefficient field $K^{\prime}$ of $\widehat{R}$, we have that

(i) $\quad \lambda_{I_{s}, \ldots, I_{1}}^{i_{s}, \ldots, i_{1}}\left(R ; K^{\prime}\right)=0$ for $i_{1}>\operatorname{dim}\left(R / I_{1}\right)$,

(ii) $\lambda_{I_{s}, \ldots, I_{1}}^{i_{s}, \ldots, i_{1}}\left(R ; K^{\prime}\right)=0$ for $i_{j}>\operatorname{dim}\left(R / I_{j-1}\right)$ and $2 \leq j \leq \ell$,

(iii) $\lambda_{I_{2}, I_{1}}^{i_{2}, i_{1}}\left(R ; K^{\prime}\right)=0$ for $i_{2}>i_{1}$

(iv) $\lambda_{I_{1}}^{i_{1}}\left(R ; K^{\prime}\right) \neq 0$ for $i_{1}=\operatorname{dim}\left(R / I_{1}\right)$, and

(v) $\lambda_{I_{2}, I_{1}}^{i_{2}, i_{1}}\left(R ; K^{\prime}\right) \neq 0$ if $i_{2}=\operatorname{dim}\left(R / I_{1}\right)-\operatorname{dim}\left(R / I_{2}\right)$ and $i_{1}=\operatorname{dim}\left(R / I_{1}\right)$.

Proof. We may assume that $R$ is complete, so that it admits a surjection $\pi: S \rightarrow R$, where $S=K\left[\left[x_{1}, \ldots, x_{n}\right]\right]$ for some $n$, and $\pi(K)=K^{\prime}$. Let $J_{j}=$ $\pi^{-1}\left(I_{j}\right)$ for $1 \leq j \leq s$.

As $S$ is Cohen-Macaulay, $\operatorname{depth}_{J_{1}}(S)=\operatorname{codim}\left(S / J_{1}\right)=n-\operatorname{dim}\left(S / J_{1}\right)=$ $n-\operatorname{dim}\left(R / I_{1}\right)$, so that (i) and (iv) hold since $H_{J_{1}}^{i_{1}}(S)=0$ if $i_{1}<\operatorname{depth}_{J_{1}}(S)$ and $H_{J_{1}}^{\operatorname{depth}_{J_{1}}(S)}(S) \neq 0$.

To see (ii), note that

$$
\begin{aligned}
\operatorname{inj.} \operatorname{dim} H_{J_{j-1}}^{i_{j-1}} \cdots H_{J_{2}}^{i_{2}} H_{J_{1}}^{n-i_{1}}(S) & \leq \operatorname{dim}\left(\operatorname{Supp} H_{J_{j-1}}^{i_{j-1}} \cdots H_{J_{2}}^{i_{2}} H_{J_{1}}^{n-i_{1}}(S)\right) \\
& \leq \operatorname{dim}\left(S / J_{j-1}\right)=\operatorname{dim}\left(R / I_{j-1}\right)
\end{aligned}
$$

by [L3]. Similarly, we have that (iii) follows because inj.dim $H_{J_{1}}^{n-i_{1}}(S) \leq$ $\operatorname{dim}\left(\operatorname{Supp} H_{J_{1}}^{n-i_{1}}(S)\right) \leq i_{1}$. 
To prove $(\mathrm{v})$, choose a minimal prime $P$ of $J_{2}$. Now, $\operatorname{Rad}\left(J_{1} S_{P}\right)=P S_{P}$ in $S_{P}$. Then $H_{P S_{P}}^{p} H_{J_{1} S_{P}}^{\operatorname{dim}\left(S_{P}\right)-q}\left(S_{P}\right) \neq 0$ when $p=q=\operatorname{dim}\left(S_{P} / J_{1} S_{P}\right)$ by [L1, Property 4.4(iii)]. Noting that

$$
\operatorname{dim}\left(S_{P}\right)=\operatorname{dim}(S)-\operatorname{dim}(S / P)=\operatorname{dim}(S)-\operatorname{dim}\left(S / J_{1}\right)=n-\operatorname{dim}\left(R / I_{1}\right),
$$

and that

$$
\operatorname{dim}\left(S_{P} / J_{1} S_{P}\right)=\operatorname{dim}\left(S / J_{1}\right)-\operatorname{dim}\left(S / J_{2}\right)=\operatorname{dim}\left(R / I_{1}\right)-\operatorname{dim}\left(R / I_{2}\right),
$$

we see that $H_{J_{2}}^{i_{2}} H_{J_{1}}^{i_{1}}(S) \otimes_{S} S_{P} \neq 0$ if $i_{2}=\operatorname{dim}\left(R / I_{1}\right)-\operatorname{dim}\left(R / I_{2}\right)$ and $i_{1}=$ $\operatorname{dim}\left(R / I_{1}\right)$.

Lemma 4.10. Given an extension of fields $K \subseteq L$, let $R=K\left[\left[x_{1}, \ldots, x_{n}\right]\right]$, and let $S=L\left[\left[x_{1}, \ldots, x_{n}\right]\right]$. Via $R \hookrightarrow S$, the map induced by the field extension, if $M$ is a simple $D(R, K)$-module, then $M \otimes_{R} S$ is a simple $D(S, L)$ module.

Proof. We have that $S=R \otimes_{K} L$ because the field extension is finite. Then $M \otimes_{R} S=M \otimes_{K} L$ and the action of $\partial \in D(S, L)$ is given by $\partial(v \otimes a)=$ $\partial(v) \otimes a$. Let $e_{1}, \ldots, e_{h}$ be a basis for $L$ as $K$-vector space. If $v \in M \otimes_{K} L$ is not zero, then $v=w_{1} \otimes e_{1}+\cdots+w_{h} \otimes e_{h}$ for some $w_{j} \in M$, where at least one $w_{j}$ is not zero. We assume that $w_{1} \neq 0$ and that there exist operators $\delta_{j} \in D(R, K)$ such that $w_{j}=\delta_{j} w_{1}$ because $M$ is simple. Let $\delta=\delta_{1}+\cdots+\delta_{h}$, and let $u=e_{1} \cdots e_{h}$. Then $v=\delta\left(w_{1} \otimes a\right)=a \delta\left(w_{1} \otimes 1\right)$. Since $v \neq 0, \delta\left(w_{1}\right) \neq 0$ and there exists $\partial \in D(S, L)$ such that $\partial \delta w_{1}=w_{1}$. Then $u^{-1} \partial v=w_{1} \otimes 1$. Therefore, for every $v \in M \otimes_{K} L$ nonzero, $v \in D(S, L) \cdot w_{1} \otimes 1$, and $w_{1} \otimes 1 \in$ $D(S, L) \cdot v$. Hence, $M \otimes_{K} L$ is a simple $D(S, L)$-module.

Proposition 4.11. Let $K \subseteq L$ be a finite field extension, and here let $R=K\left[\left[x_{1}, \ldots, x_{n}\right]\right]$, and let $S=L\left[\left[x_{1}, \ldots, x_{n}\right]\right]$. Then for all ideals $I_{1}, \ldots, I_{s}$ of $R$ and all $i_{1}, \ldots, i_{s} \in \mathbb{N}$,

$$
\lambda_{I_{s}, \ldots, I_{1}}^{i_{s}, \ldots, i_{1}}(R)=\lambda_{I_{s}, \ldots, \ldots, I_{1} S}^{i_{s}, \ldots, i_{1}}(S) .
$$

Proof. We have that $S=R \otimes_{K} L$ because the field extension is finite. Let

$$
0=M_{1} \subsetneq \cdots \subsetneq M_{\ell}=H_{I_{s}}^{i_{s}} \cdots H_{I_{2}}^{i_{2}} H_{I_{1}}^{n-i_{1}}(S)
$$

be a filtration of $D(R, K)$-modules such that each $M_{i+1} / M_{i}$ is a simple $D(R, K)$-module. Since $S$ is a faithfully flat $R$-algebra, $M_{i+1} / M_{i} \otimes_{K} L \cong$ $\left(M_{i+1} \otimes_{K} L\right) /\left(M_{i} \otimes_{K} L\right)$ is a simple $D(S, L)$-module. Thus, $\lambda_{I_{s}, \ldots, I_{1}}^{i_{s}, \ldots, i_{1}}(R)=\ell=$ $\lambda_{I_{s} S, \ldots, I_{1} S}^{i_{s}, \ldots, i_{1}}(S)$. 
Proposition 4.12. Let $I_{1}, \ldots, I_{s}$ be ideals of $S=K\left[\left[x_{1}, \ldots, x_{n}\right]\right]$, where $K$ is a field of characteristic zero. Then $\lambda_{I_{s}, \ldots, I_{1}}^{i_{s}, \ldots, i_{1}}(S) \leq e\left(H_{I_{s}}^{i_{s}} \cdots H_{I_{2}}^{i_{2}} H_{I_{1}}^{n-i_{1}}(S)\right)$, where $e(M)$ denotes $D(S, K)$-module characteristic cycle multiplicity of a holonomic $D(S, K)$-module $M$ (see Definition 2.2).

Proof. Since $H_{I_{S}}^{i_{s}} \cdots H_{I_{2}}^{i_{2}} H_{I_{1}}^{n-i_{1}}(S)$ is a holonomic $D(S, K)$-module, the claim follows from Remark 2.3.

For $R$ a 1-dimensional or complete intersection $\operatorname{ring}, \lambda_{i, j}(R)=1$ if $i=$ $j=\operatorname{dim} R$ and vanishes otherwise. However, Propositions 4.13 and 4.14 will show that the generalized Lyubeznik numbers capture finer information that can distinguish these cases.

Proposition 4.13. Let $(R, m, K)$ be a complete local ring containing a field such that $\operatorname{dim}(R)=1$. Fix a coefficient field $K^{\prime}$ of $R$, and let $P_{1}, \ldots, P_{\ell}$ denote the minimal primes of $R$. Then

$$
\lambda_{0}^{1}\left(R ; K^{\prime}\right)=\lambda_{0}^{1}\left(R / P_{1} ; K^{\prime}\right)+\cdots+\lambda_{0}^{1}\left(R / P_{\ell} ; K^{\prime}\right)+\ell-1 .
$$

Proof. By way of induction on $\ell$, first let $\ell=1$, and take a surjection $\pi: S=K\left[\left[x_{1}, \ldots, x_{n}\right]\right] \rightarrow R \cong S / I$, where $I=\operatorname{Ker}(\pi)$ and $\pi\left(K^{\prime}\right)=K$. If $P$ denotes the minimal prime of $R$, then $\pi^{-1}(P)=\operatorname{Rad}(I)$ is the only minimal prime of $I$. Then

$$
\begin{aligned}
\lambda_{0}^{1}\left(R ; K^{\prime}\right) & =\operatorname{length}_{D(S, K)} H_{I}^{n-1}(S)=\operatorname{length}_{D(S, K)} H_{\pi^{-1}(P)}^{n-1}(S) \\
& =\lambda_{0}^{1}\left(R / P ; K^{\prime}\right) .
\end{aligned}
$$

Now suppose that the formula holds for $\ell-1$. Take a surjection $\pi: S \rightarrow$ $R \cong S / I$, where $S=K\left[\left[x_{1}, \ldots, x_{n}\right]\right]$ and $\pi\left(K^{\prime}\right)=K$. Let $Q_{i}=\pi^{-1}\left(P_{i}\right)$, so that $\operatorname{Rad}(I)=Q_{1} \cap \cdots \cap Q_{\ell}$, and let $J=Q_{1} \cap \cdots \cap Q_{\ell-1}$. Since $\operatorname{Rad}\left(J+Q_{\ell}\right)=$ $\eta$, the maximal ideal of $S$, the Mayer-Vietoris sequence in local cohomology with respect to $J$ and $Q_{\ell}$ then gives the exact sequence

$$
0 \rightarrow H_{J}^{n-1}(S) \oplus H_{Q_{\ell}}^{n-1}(S) \rightarrow H_{I}^{n-1}(S) \rightarrow H_{\eta}^{n}(S) \rightarrow 0,
$$

where $H_{I+J}^{n}(S) \cong E_{S}(K)$, a simple $D(S, K)$-module (see [L2]). Then $\lambda_{0}^{1}\left(R ; K^{\prime}\right)$ equals

$$
\begin{aligned}
& \operatorname{length}_{D(S, K)} H_{I}^{n-1}(S) \\
& \quad=\operatorname{length}_{D(S, K)} H_{J}^{n-1}(S)+\operatorname{length}_{D(S, K)} H_{Q_{\ell}}^{n-1}(S)+1
\end{aligned}
$$




$$
\begin{aligned}
& =\lambda_{0}^{1}\left(S / J ; K^{\prime}\right)+\lambda_{0}^{1}\left(S / Q_{\ell} ; K^{\prime}\right)+1, \quad \text { and inductively, } \\
& =\left(\lambda_{0}^{1}\left(S / Q_{1} ; K^{\prime}\right)+\cdots+\lambda_{0}^{1}\left(S / Q_{\ell} ; K^{\prime}\right)+\ell-2\right)+\lambda_{0}^{1}\left(S / Q_{\ell} ; K^{\prime}\right)+1 \\
& =\lambda_{0}^{1}\left(R / P_{1} ; K^{\prime}\right)+\cdots+\lambda_{0}^{1}\left(R / P_{\ell} ; K^{\prime}\right)+\ell-1, \quad \text { as } R / P_{i} \cong S / Q_{i} .
\end{aligned}
$$

Proposition 4.14. Let $S=K\left[\left[x_{1}, \ldots, x_{n}\right]\right]$, where $K$ is a field. Let $f_{1}$, $\ldots, f_{\ell} \in S$ be irreducible, and let $f=f_{1}^{\alpha_{1}} \cdots f_{\ell}^{\alpha_{\ell}}, \alpha_{i} \in \mathbb{N}$. Then

$$
\lambda_{0}^{n-1}(S / f) \geq \lambda_{0}^{n-1}\left(S / f_{1}\right)+\cdots+\lambda_{0}^{n-1}\left(S / f_{\ell}\right)+\ell-1 .
$$

Proof. Since $H_{I}^{i}(S)=H_{\sqrt{I}}^{i}(S)$ for every ideal $I \subseteq S$, we may assume that $\alpha_{1}=\cdots=\alpha_{\ell}=1$. By way of induction on $\ell$, we first note that when $\ell=1$, the statement is clear. Assume that the statement holds for $\ell-1$ such elements, and let $g=f_{1} \cdots f_{\ell-1}$. Since $f_{\ell}$ and $g$ form a regular sequence, the Mayer-Vietoris sequence gives the exact sequence

$$
0 \rightarrow H_{g S}^{1}(S) \oplus H_{f_{\ell} S}^{1}(S) \rightarrow H_{f S}^{1}(S) \rightarrow H_{\left(g, f_{\ell}\right) S}^{2}(S) \rightarrow 0 .
$$

Since $H_{\left(g, f_{\ell}\right) S}^{2}(S) \neq 0$, length ${ }_{D(S, K)} H_{\left(g, f_{\ell}\right) S}^{2}(S) \geq 1$. Moreover,

$$
\begin{aligned}
\lambda_{0}^{n-1}(S / f S) \\
\quad=\operatorname{length}_{D(S, K)} H_{f S}^{1}(S) \\
\quad \geq \operatorname{length}_{D(S, K)} H_{g S}^{1}(S)+\operatorname{length}_{D(S, K)} H_{f_{\ell} S}^{1}(S)+1 \\
\quad=\lambda_{0}^{n-1}(S / g S)+\lambda_{0}^{n-1}\left(S / f_{\ell} S\right)+1, \quad \text { and inductively, } \\
\quad \geq \lambda_{0}^{n-1}\left(S / f_{1} S\right)+\cdots+\lambda_{0}^{n-1}\left(S / f_{\ell-1} S\right)+\ell-2+\lambda_{0}^{n-1}\left(S / f_{\ell} S\right)+1 \\
\quad=\lambda_{0}^{n-1}\left(S / f_{1} S\right)+\cdots+\lambda_{0}^{n-1}\left(S / f_{\ell} S\right)+\ell-1 .
\end{aligned}
$$

DEFinition 4.15 (Lyubeznik characteristic). Let $(R, m, K)$ be a local ring containing a field such that $\operatorname{dim}(R)=d$. Fix a coefficient field $K^{\prime}$ of $\widehat{R}$. The Lyubeznik characteristic of $R$ (with respect to $K^{\prime}$ ) is defined as

$$
\chi_{\lambda}\left(R ; K^{\prime}\right):=\sum_{i=0}^{d}(-1)^{i} \lambda_{0}^{i}\left(R ; K^{\prime}\right) .
$$

If the choice of the coefficient field is clear, we simply write $\chi_{\lambda}(R)$.

Proposition 4.16. Let $I$ and $J$ be ideals of a local ring $(R, m, K)$ containing a field. For any coefficient field $K^{\prime}$ of $\widehat{R}$,

$$
\chi_{\lambda}\left(R / I ; K^{\prime}\right)+\chi_{\lambda}\left(R / J ; K^{\prime}\right)=\chi_{\lambda}\left(R /(I+J) ; K^{\prime}\right)+\chi_{\lambda}\left(R / I \cap J ; K^{\prime}\right) .
$$


Proof. This is an immediate consequence of the Mayer-Vietoris sequence for local cohomology with respect to $I$ and $J$.

Proposition 4.17. Given an ideal $I=\left(f_{1}, \ldots, f_{\ell}\right)$ of $S=K\left[\left[x_{1}, \ldots, x_{n}\right]\right]$, where $K$ is a field, then

$$
\chi_{\lambda}(S / I)=(-1)^{n} \sum_{j=0}^{\ell} \sum_{1 \leq i_{1}<\cdots<i_{j} \leq \ell}(-1)^{j} \lambda_{0}^{n-1}\left(S /\left(f_{i_{1}} \cdots f_{i_{j}}\right)\right) .
$$

In particular, if $f_{1}, \ldots, f_{\ell}$ form a regular sequence or if $\operatorname{char}(K)=p>0$ and $S / I$ is a Cohen-Macaulay ring of dimension d, then $\lambda_{0}^{n-\ell}\left(S /\left(f_{1}, \ldots, f_{\ell}\right) S\right)$ (or $\lambda_{0}^{d}(S / I)$, resp. $)$ equals $\sum_{j=0}^{\ell} \sum_{1 \leq i_{1}<\cdots<i_{j} \leq \ell}(-1)^{n-d+j} \lambda_{0}^{n-1}\left(S /\left(f_{i_{1}} \cdots f_{i_{j}}\right)\right)$.

Proof. Let $D=D(S, K)$. The definition of local cohomology in terms of the Čech-like complex gives

$$
\sum_{j=0}^{\ell}(-1)^{j} \operatorname{length}_{D} H_{I}^{j}(S)=\sum_{j=0}^{\ell}(-1)^{j} \sum_{1 \leq i_{1}<\cdots<i_{j} \leq \ell} \operatorname{length}_{D} S_{f_{i_{1}} \cdot \ldots \cdot f_{i_{j}}},
$$

and the short exact sequence $0 \rightarrow S \rightarrow S_{g} \rightarrow H_{\left(f_{i_{1}} \cdot \ldots \cdot f_{i_{j}}\right)}^{1}(S) \rightarrow 0$ gives that length $_{D} S_{f_{i_{1}} \cdot \ldots \cdot f_{i_{j}}}=$ length $_{D} H_{\left(f_{i_{1}} \cdot \ldots \cdot f_{i_{j}}\right)}^{1}+1$. The first statement then follows from a straightforward calculation. The statement for a regular sequence is an immediate consequence, and the final statement follows since the only nonvanishing local cohomology module is $H_{I}^{n-d}(S)$ by [PS, Proposition 4.1], since $S / I$ is Cohen-Macaulay.

The following two remarks indicate that the generalized Lyubeznik numbers of the form $\lambda_{0}^{d}(R)$, where $\operatorname{dim} R=d$, measure singularities in characteristic $p>0$.

REmark 4.18. Blickle's results [Bl, Theorem 4.9, Corollaries 4.10 and 4.16] indicate that the generalized Lyubeznik numbers detect $F$-regularity and $F$-rationality. Let $(R, m, K)$ be a complete local domain of characteristic $p>0$ and of dimension $d$, such that $K$ is $F$-finite. For any coefficient field $K^{\prime}$ of $R$, the following hold.

- If $\lambda_{0}^{d}\left(R ; K^{\prime}\right)=1$, then $0_{H_{m}^{d}(R)}^{*}$ is $F$-nilpotent.

- If $R$ is $F$-injective and $\lambda_{0}^{d}\left(R ; K^{\prime}\right)=1$, then $R$ is $F$-rational.

If $K$ is additionally assumed to be perfect, the following also hold.

- We have $\lambda_{0}^{d}(R)=1$ if and only if $0_{H_{m}^{d}(R)}^{*}$ is $F$-nilpotent.

- If $R$ is $F$-rational, then $\lambda_{0}^{d}(R)=1$. 
- If $R$ is $F$-injective, then $\lambda_{0}^{d}(R)=1$ if and only if $R$ is $F$-rational.

If, in addition, $R$ is 1-dimensional, the following hold.

- If $\lambda_{0}^{d}\left(R ; K^{\prime}\right)=1$, then $R$ is unibranch.

- If $K$ is perfect, then $\lambda_{0}^{d}(R)=1$ if and only if $R$ is unibranch.

REMARK 4.19. In certain cases, the generalized Lyubeznik numbers may measure how "far" an $F$-pure ring is from being $F$-regular. Take an element $f$ of an $F$-finite regular local ring $S$ of characteristic $p>0$ such that the quotient ring $S / f S$ is $F$-pure. As demonstrated by Vassilev [V, Corollary 3.4], if $\tau_{1}$ denotes the pullback of the test ideal of $S / f S$ to $S$, and if, for $i>1$, $\tau_{i}$ is the pullback of the test ideal of the ring $S / \tau_{i-1}$ to $S$, then we have a chain of ideals

$$
(f) \subsetneq \tau_{1} \subsetneq \tau_{1} \subsetneq \cdots \subsetneq \tau_{\ell}=S
$$

where each quotient $S / \tau_{i}$ is $F$-pure. The first author and Pérez [NP] have shown that $\lambda_{0}^{d}\left(S / f S ; K^{\prime}\right) \geq \ell$, where $d=\operatorname{dim}(S / f S)$. Thus, $S / f S$ must be $F$-regular if $\lambda_{0}^{d}\left(S / f S ; K^{\prime}\right)=1$, and a large value for $\lambda_{0}^{d}\left(S / f S ; K^{\prime}\right)$ may indicate that $S / f S$ is "far" from being $F$-regular.

\section{$\S 5$. (Generalized) Lyubeznik numbers of ideals generated by max- imal minors}

We begin this section by illustrating how the behavior of the Lyubeznik numbers may differ in the local and global cases. Using this observation, we give an example in which the generalized Lyubeznik numbers depend on the characteristic (see Example 5.4).

Lemma 5.1. Suppose that $K$ is a field of characteristic zero, that $R=$ $K\left[x_{1}, \ldots, x_{n}\right]$, and that $S=K\left[\left[x_{1}, \ldots, x_{n}\right]\right]$. Take a homogeneous polynomial $f \in R$. If for some $N \in \mathbb{N}, D(S, K) \cdot 1 / f^{N}=S_{f}$, then $D(R, K) \cdot 1 / f^{N}=R_{f}$.

Proof. Let $D_{R}=D(R, K)$, and let $D_{S}=D(S, K)$. For every $r \in \mathbb{N}$, there exists $\delta=\sum_{\alpha} g_{\alpha} \frac{\partial^{\alpha}}{\partial x^{\alpha}} \in D_{S}=S\left\langle\frac{\partial}{\partial x_{1}}, \ldots, \frac{\partial}{\partial x_{n}}\right\rangle$ such that $\delta\left(1 / f^{N}\right)=1 / f^{r}$. In addition, there exist $\mu \in \mathbb{N}$ and homogeneous $h_{\alpha} \in R$ such that $\mu>r$ and $\frac{\partial^{\alpha}}{\partial x^{\alpha}} \frac{1}{f^{N}}=h_{\alpha} / f^{\mu}$, so $\delta\left(1 / f^{N}\right)=\sum_{\alpha} g_{\alpha}\left(h_{\alpha} / f^{\mu}\right)=1 / f^{r}$.

We have that $\sum_{\alpha} g_{\alpha} h_{\alpha}=f^{\mu-r}$, and there exist homogeneous $g_{\alpha, t} \in R$ of degree $t$ such that $g_{\alpha}=\sum_{t=0}^{\infty} g_{\alpha, t}$. If $t_{\alpha}=(\mu-r) \operatorname{deg}(f)-\operatorname{deg}\left(h_{\alpha}\right)$, then

$$
f^{\mu-r}=\sum_{\alpha} g_{\alpha} h_{\alpha}=\sum_{\alpha} \sum_{t=0}^{\infty} g_{\alpha, t} h_{\alpha}=\sum_{\alpha} g_{\alpha, t_{\alpha}} h_{\alpha}
$$

because $f$ and $h_{\alpha}$ are homogeneous polynomials. 
Let $\widetilde{\delta}=\sum_{\alpha} g_{\alpha, t_{\alpha}} \frac{\partial^{\alpha}}{\partial x^{\alpha}} \in D_{R}$. Then

$$
\widetilde{\delta} \cdot \frac{1}{f^{N}}=\sum_{\alpha} g_{\alpha, t_{\alpha}} \frac{\partial^{\alpha}}{\partial x^{\alpha}} \frac{1}{f^{N}}=\sum_{\alpha} g_{\alpha, t_{\alpha}} \frac{h_{\alpha}}{f^{\mu}}=\frac{\sum_{\alpha} g_{\alpha, t_{\alpha}} h_{\alpha}}{f^{\mu}}=\frac{f^{\mu-r}}{f^{\mu}}=\frac{1}{f^{r}} .
$$

Hence, $1 / f^{r} \in D_{R} \cdot 1 / f^{N}$, and the result follows.

REMARK 5.2. The conclusion of Lemma 5.1 is not necessarily true if $f$ is not a homogeneous polynomial. Indeed, let $m$ denote the homogeneous maximal ideal of $R$. If $f \in R$ is any polynomial such that $R_{m} / f R_{m}$ is a regular local ring, then even if $D(R, K) \cdot 1 / f^{N} \neq R_{f}$, we have that $D(S, K) \cdot 1 / f=S_{f}$.

Fix $f \in R=K\left[x_{1}, \ldots, x_{n}\right]$. If $s$ denotes an indeterminate, there exist $b(s) \in K[s]$ and $\delta(s) \in D[s]=K[s] \otimes_{K} D$ such that for all $\ell \in \mathbb{Z}$,

$$
b(\ell) f^{\ell}=\delta(\ell) \cdot f^{\ell+1} .
$$

All such $b(s) \in K[s]$ satisfying (5.2.1) form an ideal of $K[s]$. The unique monic generator of this ideal is denoted $b_{f}(s)$ and is called the BernsteinSato polynomial of $f$ [Co, Chapter 10].

REMARK 5.3. Let $b_{f}(s)$ denote the Bernstein-Sato polynomial of $f \in$ $R=K\left[x_{1}, \ldots, x_{n}\right]$ over $R$. If $N=\max \left\{j \in \mathbb{N} \mid b_{f}(-j)=0\right\}$, then we have $D(R, K) \cdot 1 / f^{N-1} \neq R_{f}$ (see [W2, Lemma 1.3]). Therefore, if $f \in R$ is homogeneous, length $_{D(S, K)} H_{(f)}^{1}(S) \geq 2$ by Lemma 5.1 .

EXAMPLE 5.4. Let $R=K[X]$ be the polynomial ring over a field $K$ in the entries of an $r \times r$ matrix $X$ of indeterminates, and let $\operatorname{det}(X)$ denote the determinant of $X$. If $K$ is a perfect field of characteristic $p>0$, $R /(\operatorname{det}(X))$ is $F$-rational (see [HH, Theorem 7.14]), so by Remark 4.18, $\lambda_{0}^{r^{2}-1}\left(R_{m} / \operatorname{det}(X) R_{m}\right)=1$. In contrast, if $K$ has characteristic zero, the Bernstein-Sato polynomial of the determinant of $X$ over $R$ is $b_{\operatorname{det}(X)}(s)=$ $(s+1)(s+2) \cdots(s+r)$, so by Remark 5.3, $\lambda_{0}^{r^{2}-1}\left(R_{m} / \operatorname{det}(X) R_{m}\right) \geq 2$. In particular, even when a generalized Lyubeznik number is nonzero in both characteristic zero and characteristic $p>0$, its values may differ.

Now we study the case when the matrix is not square in characteristic zero.

Notation 5.5. Let $R$ be the polynomial ring over a field $K$ of characteristic zero in the entries of $X=\left[x_{i j}\right]$, an $r \times s$ matrix of indeterminates, 
where $r<s$. Let $m$ denote its homogeneous maximal ideal, let $I_{t}$ be the ideal generated by the $t \times t$ minors of $X$, and let $I=I_{r}$ be the ideal generated by the maximal minors of $X$.

EXAMPLE 5.6. Under Notation 5.5, $H_{I}^{r(s-r)+1}(R) \cong E_{R}(K), \quad 0 \neq$ $H_{I}^{i}(R) \hookrightarrow H_{I}^{i}(R)_{I_{t+1}} \cong E_{R}\left(R / I_{t+1}\right)$ for $i=(r-t)(s-r)+1,0<t<r$, and all other $H_{I}^{i}(R)=0$ (see [Wi, Theorem 1.1]). In particular, $\lambda_{0}^{j}\left(R_{m} / I R_{m}\right)$ equals one for $j=r^{2}-1$ and vanishes if $j \neq r^{2}+t(s-r)-1$ for some $0 \leq t<r$. Moreover, $\lambda_{0, j}\left(R_{m} / I R_{m}\right)$ equals one if $j=r^{2}-1$ and vanishes otherwise.

We now compute the Lyubeznik numbers $\lambda_{i, j}\left(R_{m} / I R_{m}\right)$ in a special case.

Proposition 5.7. Under Notation 5.5, if $r=2$, then

$$
\lambda_{0,3}\left(R_{m} / I R_{m}\right)=\lambda_{s-1, s+1}\left(R_{m} / I R_{m}\right)=\lambda_{s+1, s+1}\left(R_{m} / I R_{m}\right)=1,
$$

and all other $\lambda_{i, j}\left(R_{m} / I R_{m}\right)=0$.

Proof. By Example 5.6, the only two nonzero local cohomology modules $H_{I}^{i}(R)$ are $H_{I}^{2 s-3}(R) \cong E_{R}(K)$ and $H_{I}^{s-1}(R) \hookrightarrow E_{R}(R / I)$. Replace $R$ by its localization at $m$, and consider the spectral sequence $E_{2}^{p, q}=H_{m}^{p} H_{I}^{q}(R) \Longrightarrow$ $H_{m}^{p+q}(R)=E_{\infty}^{p, q}\left(\right.$ see [Ha, Proposition 1.4]). Now, $H_{m}^{0} H_{I}^{2 s-3}(R) \cong E_{R}(K)$ and $H_{m}^{p} H_{I}^{2 s-3}(R)=0$ for $p>0$; in particular, $\lambda_{0,3}(R / I)=1$. Also note that $\operatorname{dim} R / I=s+1$, so that $H_{m}^{p} H_{I}^{s-1}(R)=0$ for $p>s+1$. These observations are incorporated in Figure 1.

The only possibly nonzero $E_{2}^{p, q}=H_{m}^{p} H_{I}^{q}(R)$ such that $p+q=\operatorname{dim}(R)$ is $E_{2}^{s+1, s-1}$. Since the spectral sequence maps to and from the $(s+1, s-1)$ spot must all be zero, we must have that $H_{m}^{s+1} H_{I}^{s-1}(R) \cong E_{\infty}^{s+1, s-1} \cong E_{R}(K)$, so that $\lambda_{s+1, s+1}(R / I)=1$ (and all other $E_{\infty}^{p, q}=0$ ).

The sole differential that is possibly nonzero is $d_{s-1}^{0,2 s-3}: E_{s-1}^{0,2 s-3} \cong$ $E_{R}(K) \rightarrow E_{s-1}^{s-1, s-1}$. After taking cohomology with respect to the $d_{s-1}^{p, q}$, we must get zero at both the $(s-1, s-1)$ and $(0,2 s-3)$ spots, so $d_{s-1}^{0,2 s-3}$ must be an isomorphism, and $H_{m}^{s-1} H_{I}^{s-1}(R)=E_{s-1}^{s-1, s-1} \cong E_{R}(K)$, so that $\lambda_{s-1, s+1}(R / I)=1$. Since all other maps are zero, and after taking cohomology with respect to $d_{s-1}^{p, q}$ we must also get zero, all remaining local cohomology modules $H_{I}^{p} H_{m}^{q}(R)=0$, so that $\lambda_{p, q}(R / I)=0$.

Corollary 5.8. With Notation 5.5, let $r=2$. As $H_{I}^{s-1}(R) \hookrightarrow H_{I}^{s-1}(R)_{I}$ by Example 5.6, we also have natural injections $H_{I}^{s-1}(R)_{x_{i j}} \hookrightarrow H_{I}^{s-1}(R)_{I}$ 


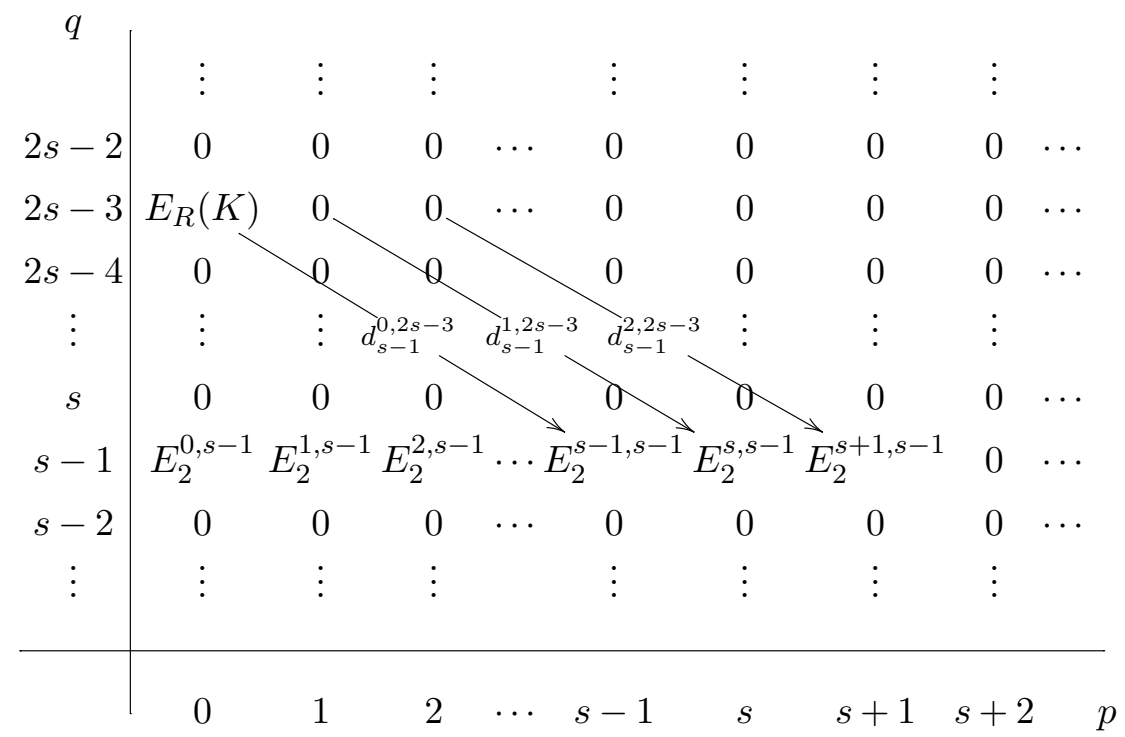

Figure 1: $E_{2}^{p, q}=H_{m}^{p} H_{I}^{q}(R)$.

for each indeterminate $x_{i j}$. Under this identification,

$$
H_{I}^{s-1}(R) \cong \bigcap_{\substack{i=1, \ldots, r \\ j=1,2}} H_{I}^{s-1}(R)_{x_{i j}} \subseteq H_{I}^{s-1}(R)_{I} .
$$

Proof. Let $C$ denote the cokernel of the initial injection, so that we have the long exact sequence

$$
\begin{aligned}
0 & \rightarrow H_{m}^{0} H_{I}^{s-1}(R) \rightarrow H_{m}^{0}\left(H_{I}^{s-1}(R)_{I}\right) \rightarrow H_{m}^{0}(C) \rightarrow H_{m}^{1} H_{I}^{s-1}(R) \\
& \rightarrow H_{m}^{1}\left(H_{I}^{s-1}(R)_{I}\right) \rightarrow \cdots .
\end{aligned}
$$

Since $I$ is the only associated prime of $H_{I}^{s-1}(R)$ and of $H_{I}^{s-1}(R)_{I} \cong$ $E_{R}(R / I)$, it follows that $H_{m}^{0} H_{I}^{s-1}(R)=H_{m}^{0}\left(H_{I}^{s-1}(R)_{I}\right)=H_{m}^{1}\left(H_{I}^{s-1}(R)_{I}\right)=$ 0 , so that $H_{m}^{0}(C) \cong H_{m}^{1} H_{I}^{s-1}(R)$, which vanishes by Proposition 5.7.

Noting that the injections $H_{I}^{s-1}(R)_{x_{i j}} \hookrightarrow H_{I}^{s-1}(R)_{I}$ are induced since the only associated prime of $H_{I}^{s-1}(R)$ is $I$ and $x_{i j} \notin I$, let $M=$ $\bigcap_{1<i<r, 1<j<2} H_{I}^{s-1}(R)_{x_{i j}} \subseteq H_{I}^{s-1}(R)_{I}$ under the identification. Then $M / H_{I}^{i}(R)$ injects into $H_{I}^{s-1}(R)_{I} / H_{I}^{s-1}(R)=C$. Moreover, since every element of $M / H_{I}^{i}(R)$ is killed by a power of $m, M / H_{I}^{i}(R)=H_{m}^{0}\left(M / H_{I}^{i}(R)\right) \hookrightarrow$ $H_{m}^{0}(C)=0$. Thus, $M=H_{I}^{s-1}(R)$. 


\section{§6. Generalized Lyubeznik numbers of monomial ideals}

In this section we characterize the generalized Lyubeznik numbers associated to monomial ideals. To do so, we make use of the categories of squarefree and straight modules introduced by Yanagawa ([Y1], [Y2]); we begin with some definitions and notation he first introduced.

Notation 6.1. Let $S=K\left[x_{1}, \ldots, x_{n}\right], K$ a field, and consider the natural $\mathbb{N}^{n}$-grading on $S$. For $\alpha=\left(\alpha_{1}, \ldots, \alpha_{n}\right) \in \mathbb{Z}^{n}$, we define $\operatorname{Supp}(\alpha)=\{i \mid$ $\left.\alpha_{i}>0\right\} \subseteq[n]=\{1, \ldots, n\}$. For a monomial $x^{\alpha}=x_{1}^{\alpha_{1}} \cdots x_{n}^{\alpha_{n}}, \operatorname{Supp}\left(x^{\alpha}\right):=$ $\operatorname{Supp}(\alpha)$. We say that $x^{\alpha}$ is square-free if, for every $i \in[n], \alpha_{i} \in\{0,1\}$. Let $e_{i}$ denote the vector $(0, \ldots, 0,1,0, \ldots, 0) \in \mathbb{N}^{n}$, where " 1 " is in the $i$ th entry. If $F \subseteq[n]$, let $P_{F}$ denote the prime ideal generated by $\left\{x_{i} \mid i \notin F\right\}$. If $F \subseteq[n]$, we will often use $F$ to denote $\sum_{i \in F} e_{i}$; for instance, $x^{F}$ denotes $\prod_{i \in F} x_{i}$.

Given a $\mathbb{Z}^{n}$-graded $S$-module $M$ and $\beta \in \mathbb{Z}, M(\beta)$ denotes the $\mathbb{N}^{n}$-graded $S$-module that has underlying $S$-module $M$ but with a shift in the grading: $M(\beta)_{\alpha}=M_{\alpha+\beta}$. Let $\omega_{S}=S(-1, \ldots,-1)$ denote the canonical module of $S$, and let $*$ Mon denote the category of $\mathbb{Z}^{n}$-graded $S$-modules.

Definition 6.2 (Square-free monomial module). An $\mathbb{N}^{n}$-graded $S$-module $M=\bigoplus_{\beta \in \mathbb{N}^{n}} M_{\beta}$ is square-free if it is finitely generated, and the multiplication map $M_{\alpha} \stackrel{\cdot x_{i}}{\longrightarrow} M_{\alpha+e_{i}}$ is bijective for all $\alpha \in \mathbb{N}$ and all $i \in \operatorname{Supp}(\alpha)$. The category of square-free $S$-modules is denoted $\mathbf{S q}$, a subcategory of $*$ Mon.

If $I$ is a square-free monomial ideal, then both $I$ and $S / I$ are square-free modules. Moreover, if $0 \rightarrow M^{\prime} \rightarrow M \rightarrow M^{\prime \prime} \rightarrow 0$ is a short exact sequence in *Mon, then $M$ is a square-free module if and only if both $M^{\prime}$ and $M^{\prime \prime}$ are square-free modules. In addition, if $M$ is a square-free module, then $\operatorname{Ext}_{S}^{i}\left(M, \omega_{S}\right)$ is a square-free module for every $i \in \mathbb{N}$ (see [Y1, Theorem 2.6]). Moreover, for any subset $G \subseteq F \subseteq[n], S / P_{F}(-G)$ is a square-free module (where the grading of $P_{F}(-G)$ satisfies $\left[P_{F}(-G)\right]_{\ell}=\left[P_{F}\right]_{\ell-G}$ ).

REMARK 6.3. An $\mathbb{N}^{n}$-graded square-free $S$-module $M$ is a simple squarefree module if it has no proper nontrivial square-free submodules. In fact, such a square-free module is simple if and only if it is isomorphic to $S$ / $P_{F}(-F)$ for some $F \subseteq[n]$.

Proposition 6.4 ([Y1, Proposition 2.5]). An $\mathbb{N}^{n}$-graded $S$-module $M$ is square-free if and only if there exists a filtration of $\mathbb{N}^{n}$-graded submodules $0=M_{0} \subsetneq M_{1} \subsetneq \cdots \subsetneq M_{t}=M$ such that, for each $i, 0 \leq i \leq t-1, \bar{M}_{i}=$ 
$M_{i} / M_{i+1} \cong S / P_{F_{i}}\left(-F_{i}\right)$ for some $F_{i} \subseteq[n]$ (and so is, in particular, a simple square-free module).

As a consequence of Proposition 6.4, every square-free module $M$ has finite length in Sq. We now recall the following definition (see [Y2]).

Definition 6.5 ([Y2, Definition 2.6] Straight module). A $\mathbb{Z}^{n}$-graded $S$-module $M=\bigoplus_{\beta \in \mathbb{Z}^{n}} M_{\beta}$ is straight if $\operatorname{dim}\left(M_{\beta}\right)<\infty$ for all $\beta \in \mathbb{Z}^{n}$ and the multiplication map $M_{\alpha} \stackrel{\cdot x_{i}}{\longrightarrow} M_{\alpha+e_{i}}$ is bijective for all $\alpha \in \mathbb{Z}^{n}$ and all $i \in \operatorname{Supp}(\alpha)$. The category of straight $S$-modules is denoted $\mathbf{S t r}$, a subcategory of *Mon.

REMARK 6.6. If $M=\bigoplus_{\beta \in \mathbb{Z}^{n}} M_{\beta}$ is a straight module, then $\bar{M}$ denotes the $\mathbb{N}^{n}$-graded (square-free) submodule $\bigoplus_{\beta \in \mathbb{N}^{n}} M_{\beta}$. On the other hand, if $M$ is a square-free module, we can define the straight hull of $M, \widetilde{M}$, as follows. For $\alpha \in \mathbb{N}^{n}$, let $\widetilde{M}_{\alpha}$ be a vector space isomorphic to $M_{\operatorname{Supp}(\alpha)}$, and let $\phi_{\alpha}: \widetilde{M}_{\alpha} \rightarrow M_{\operatorname{Supp}(\alpha)}$ denote such an isomorphism. Let $\beta=\alpha+e_{i}$ for some $i \in[n]$. If $\operatorname{Supp}(\alpha)=\operatorname{Supp}(\beta)$, we define $\widetilde{M}_{\alpha} \stackrel{\cdot x_{i}}{\longrightarrow} \widetilde{M}_{\beta}$ by the composition $\widetilde{M}_{\alpha} \stackrel{\phi_{\alpha}}{\longrightarrow} M_{\operatorname{Supp}(\alpha)} \stackrel{\phi_{\beta}^{-1}}{\longrightarrow} \widetilde{M}_{\beta}$; otherwise, we define $\widetilde{M}_{\alpha} \stackrel{\cdot x_{i}}{\longrightarrow} \widetilde{M}_{\beta}$ by the composition $\widetilde{M}_{\alpha} \stackrel{\phi_{\alpha}}{\longrightarrow} M_{\operatorname{Supp}(\alpha)} \stackrel{x_{i}}{\longrightarrow} M_{\operatorname{Supp}(\beta)} \stackrel{\phi_{\beta}^{-1}}{\longrightarrow} \widetilde{M}_{\beta}$. Then $\widetilde{M}$ is straight, and its $\mathbb{N}^{n}$-graded part is isomorphic to $M$.

Proposition 6.7 ([Y2, Proposition 2.7]). Continuing with the notation above, the functor $\mathbf{S t r} \rightarrow \mathbf{S q}$ defined by $M \rightarrow \bar{M}$ is an equivalence of categories with inverse functor $N \rightarrow \widetilde{N}$.

Remark 6.8. Let $L[F]$ denote the straight hull of $P_{F}(-F)$. By Proposition 6.7 (noting Remark 6.3), $L[F]$ is a simple straight module. We have that $L[F]_{\alpha}=k$ if $\operatorname{Supp}(\alpha)=F$ and is zero otherwise (see [Y2, p. 48]). Thus, $L[F] \cong H_{P_{F}}^{\ell}\left(\omega_{S}\right)$, where $\ell=n-|F|$.

REMARK 6.9. Any straight module $M$ may be given the structure of a $D(S, K)$-module. Indeed, it suffices to define an action of $\frac{1}{t !} \frac{\partial^{t}}{\partial x_{i}{ }^{t}}$ for every $1 \leq$ $i \leq n$ and $t \geq 1$. Take $v \in M_{\alpha}$, where $\alpha=\left(\alpha_{1}, \ldots, \alpha_{n}\right)$. If $1 \leq \alpha_{i} \leq t$, we define $\frac{1}{t !} \frac{\partial^{t}}{\partial x_{i} t} v=0$. Otherwise, there exists $w \in M_{\alpha-t e_{i}}$ such that $x_{i}^{t} w=v$, and we define $\frac{1}{t !} \frac{\partial^{t}}{\partial x_{i}{ }^{t}} v=\left(\begin{array}{c}\alpha_{i} \\ t\end{array}\right) w$ if $\alpha_{i}>0$ and we define $\frac{1}{t !} \frac{\partial^{t}}{\partial x_{i}{ }^{t}} v=(-1)^{-\alpha_{i}+1}\left(\begin{array}{c}-\alpha_{i} \\ t\end{array}\right) w$ if $\alpha_{i}<0$. This fact is noted in [Y2, Remark 2.12] in a specific setting, but it holds for any field. We note that this $D(S, K)$-structure gives an exact faithful functor from Str to the category of $D(S, K)$-modules. 
TheOrem 6.10. Let $K$ be a field, let $S=K\left[x_{1}, \ldots, x_{n}\right]$, and let $\widehat{S}=$ $K\left[\left[x_{1}, \ldots, x_{n}\right]\right]$. Let $I_{1}, \ldots, I_{s} \subseteq S$ be ideals generated by square-free monomials. Then

$$
\begin{aligned}
\lambda_{I_{s}, \ldots, I_{1}}^{i_{s}, \ldots, i_{1}}(\widehat{S}) & =\text { length }_{\mathbf{S t r}} H_{I_{s}}^{i_{s}} \cdots H_{I_{2}}^{i_{2}} H_{I_{1}}^{n-i_{1}}\left(\omega_{S}\right) \\
& =\sum_{\alpha \in\{0,1\}^{n}} \operatorname{dim}_{k}\left[H_{I_{s}}^{i_{s}} \cdots H_{I_{2}}^{i_{2}} H_{I_{1}}^{n-i_{1}}\left(\omega_{S}\right)\right]_{-\alpha} .
\end{aligned}
$$

Moreover, if $\operatorname{char}(K)=0$, then $\lambda_{I_{1}, \ldots, I_{s}}^{i_{1}, \ldots, i_{s}}(\widehat{S})=e\left(H_{I_{s}}^{i_{s}} \cdots H_{I_{2}}^{i_{2}} H_{I_{1}}^{n-i_{1}}(S)\right)$, where $e(M)$ denotes $D(S, K)$-module characteristic cycle multiplicity of a holonomic $D(S, K)$-module $M$ (see Definition 2.2).

Proof. In this case, we let $M=H_{I_{s}}^{i_{s}} \cdots H_{I_{2}}^{i_{2}} H_{I_{1}}^{n-i_{1}}(S)$, so that $\lambda_{I_{1}, \ldots, I_{s}}^{i_{1}, \ldots, i_{s}}(\widehat{S})=$ length $_{D(\widehat{S}, K)} M$. By applying [Y2, Corollary 3.3] iteratively, we see that $H_{I_{s}}^{i_{s}} \cdots H_{I_{2}}^{i_{2}} H_{I_{1}}^{n-i_{1}}\left(\omega_{S}\right)$ is an straight module. By Propositions 6.4 and 6.7, there is a strict ascending filtration of $\mathbb{N}^{n}$-graded submodules $0=M_{0} \subsetneq M_{1} \subsetneq \cdots \subsetneq M_{t}=M$ such that each quotient $M_{i} / M_{i+1}$ is isomorphic to $\left.\widehat{P_{F_{i}}\left(-F_{i}\right.}\right) \cong H_{P_{F_{i}}}^{n-\left|F_{i}\right|}\left(\omega_{S}\right)$, and is also a filtration of $D(S, K)$-modules by Remark 6.9. Moreover,

$$
0=M_{0} \otimes_{S} \widehat{S} \subsetneq M_{1} \otimes_{S} \widehat{S} \subsetneq \cdots \subsetneq M_{t} \otimes_{S} \widehat{S}=M \otimes_{S} \widehat{S}
$$

is a filtration of $D(\widehat{S}, K)$-modules such that $\left(\widetilde{M}_{i} \otimes_{S} \widehat{S}\right) /\left(\widetilde{M}_{i-1} \otimes_{S} \widehat{S}\right) \cong$ $\left.\widehat{P_{F_{i}}\left(-F_{i}\right.}\right) \otimes_{S} \widehat{S} \cong H_{P_{F_{i}}}^{n-\left|F_{i}\right|}(\widehat{S})$. Since $H_{P_{F_{i}}}^{n-\left|F_{i}\right|}(\widehat{S})$ is a simple $D(\widehat{S}, K)$-module for every $F \subseteq[n]$, length ${ }_{D(\widehat{S}, K)} M \otimes_{S} \widehat{S}=t$ as well.

If $K$ has characteristic zero, due to the filtration above and noting Remark 2.3,

$$
C C(M)=\sum_{i=1}^{t} C C\left(\widetilde{M}_{i} / \widetilde{M}_{i-1}\right)=\sum_{i=1}^{t} C C\left(H_{P_{F_{i}}}^{n-\left|F_{i}\right|}(S)\right)
$$

(see Definition 2.2). By [A1, Corollary 3.3 and Remark 3.4], each $C C\left(H_{P_{F_{i}}}^{n-\left|F_{i}\right|}\right)=T_{\left\{x_{i}=0 \mid x_{i} \in P_{F_{i}}\right\}}^{*} \operatorname{Spec}(S)$. As a result, each $e\left(H_{P_{F}}^{n-|F|}\right)=1$, and so $e(M)=t$. Then $\lambda_{I_{1}, \ldots, I_{s}}^{i_{1}, \ldots, i_{s}}(\widehat{S})=$ length $_{\mathbf{S q}} \bar{M}=$ length $_{\mathbf{S t r}} M=e(M)$.

REMARK 6.11. The Lyubeznik numbers with respect to monomial ideals may depend on the field, as shown in [AV, Example 4.6]. 
REMARK 6.12. For $K$ a field of characteristic zero, let $S=K\left[x_{1}, \ldots, x_{n}\right]$, and take $I \subseteq S$ an ideal generated by monomials. Let $\widehat{S}=K\left[\left[x_{1}, \ldots, x_{n}\right]\right]$. Combining work of Álvarez Montaner [A1, Theorem 3.8 and Algorithm 1] with Theorem 6.10 provides an algorithm to compute $\lambda_{0}^{j}(\widehat{S} / I \widehat{S})$ in terms of $P_{1}, \ldots, P_{N}$, the minimal primes of $I$. A consequence of this algorithm is the following inequality:

$$
\lambda_{0}^{j}(\widehat{S} / I \widehat{S}) \leq \sum_{1 \leq i_{1}<\cdots<i_{\ell}<N} \delta_{i_{1}, \ldots, i_{\ell}}^{n-j}
$$

where $\delta_{i_{1}, \ldots, i_{\ell}}^{r}=1$ if height $\left(P_{i_{1}}+\cdots+P_{i_{\ell}}\right)=r+\ell-1$ and equals zero otherwise.

REMARK 6.13. By Remark 6.12, there is a straightforward algorithm to compute the $\lambda_{0}^{i}(\widehat{S} / I \widehat{S})$ using the minimal primes of $I$.

Lemma 6.14. Let $S=K\left[\left[x_{1}, \ldots, x_{n}\right]\right], K$ a field. For a monomial $f$ with $|\operatorname{Supp}(f)|=j, \operatorname{length}_{D(S, K)} S_{f}=2^{j}$.

Proof. By 6.10, length ${ }_{D(S, K)} H_{\left(x_{i_{1}} \cdot \ldots \cdot x_{i_{j}}\right)}^{1}(S)=2^{j}-1$. Since local cohomology depends only on the radical of an ideal, $H_{f}^{1}(S)=H_{\left(x_{i_{1}} \cdot \ldots \cdot x_{i_{j}}\right)}^{1}(S)=$ $2^{j}-1$. Because of the exact sequence $0 \rightarrow S \rightarrow S_{f} \rightarrow H_{f}^{1}(S) \rightarrow 0$ and the fact that $S$ is a simple $D(S, K)$-module, we have that $\operatorname{length}_{D(S, K)} S_{f}=$ length $_{D(S, K)} S+\operatorname{length}_{D(S, K)} H_{f}^{1}(S)=2^{j}$.

Proposition 6.15. Let $K$ be a field, let $S=K\left[\left[x_{1}, \ldots, x_{n}\right]\right]$, and let $I$ be an ideal of $S$ generated by square-free monomials $f_{1}, \ldots, f_{\ell} \in S$. Then

$$
\chi_{\lambda}(S / I)=(-1)^{n} \sum_{j=0}^{\ell} \sum_{1 \leq i_{1}<\cdots<i_{j} \leq \ell}(-1)^{j} 2^{\operatorname{deg} \operatorname{lcm}\left(f_{i_{1}}, \ldots, f_{i_{j}}\right)} .
$$

Moreover, if $S / I$ is also Cohen-Macaulay, the above equation equals $(-1)^{d} \lambda_{0}^{d}(S / I)$. If, further, $f_{1}, \ldots, f_{\ell}$ form a regular sequence, this equals $(-1)^{n-1} \prod_{i=1}^{\ell}\left(2^{\operatorname{deg} f_{i}}-1\right)^{\ell}$.

Proof. Since $\left|\operatorname{Supp}\left(f_{i_{1}} \cdot \ldots \cdot f_{i_{j}}\right)\right|=\operatorname{deg} \operatorname{lcm}\left(f_{i_{1}}, \ldots, f_{i_{j}}\right)$, the first statement follows from Lemma 6.14 and Proposition 4.17.

If $S / I$ is Cohen-Macaulay, then by [A1, Proposition 3.1] (which is stated in characteristic zero, although the argument is characteristic independent), $H_{I}^{j}(S)=0$ for all $j \neq \mathrm{ht} I=n-d$, and the statement follows. If the $f_{i}$ 
also form a regular sequence, $\operatorname{lcm}\left(f_{i_{1}} \cdots f_{i_{j}}\right)=f_{i_{1}} \cdots f_{i_{j}}$ and $\operatorname{deg}\left(f_{i_{1}} \cdots f_{i_{j}}\right)=$ $\sum_{r=1}^{j} \operatorname{deg} f_{i_{r}}$, and

$$
\begin{aligned}
\sum_{j=0}^{\ell} \sum_{1 \leq i_{1}<\cdots<i_{j} \leq \ell}(-1)^{j} 2^{\left(\sum_{r=1}^{j} \operatorname{deg} f_{i_{r}}\right)} & =\prod_{i=1}^{\ell}\left(1-2^{\operatorname{deg} f_{i}}\right)^{\ell} \\
& =-\prod_{i=1}^{\ell}\left(2^{\operatorname{deg} f_{i}}-1\right)^{\ell} .
\end{aligned}
$$

\subsection{Lyubeznik characteristic of Stanley-Reisner rings}

DEFinition 6.16 (Simplicial complex, faces/simplices, dimension of a face, $i$-face, facet). A simplicial complex $\Delta$ on the vertex set $[n]=\{1, \ldots, n\}$ is a collection of subsets, called faces or simplices, that are closed under taking subsets. A face $\sigma \in \Delta$ of cardinality $|\sigma|=i+1$ is said to have dimension $i$ and is called an $i$-face of $\Delta$. The dimension of $\Delta, \operatorname{dim}(\Delta)$, is the maximum of the dimensions of its faces (or $-\infty$ if $\Delta=\varnothing$ ). We denote the set of faces of dimension $i$ of $\Delta$ by $F_{i}(\Delta)$. A face is a facet if it is not contained in any other face.

REMARK 6.17. If $\Delta_{1}$ and $\Delta_{2}$ are simplicial complexes on the vertex set $[n]$, then $\Delta_{1} \cap \Delta_{2}$ and $\Delta_{1} \cup \Delta_{2}$ are also simplicial complexes.

Definition 6.18 (Simple simplicial complex). We say that a simplicial complex $\Delta$ on the vertex set $[n]$ is simple if it is equal to $\mathcal{P}(\sigma)$, the power set of a subset $\sigma$ of $[n]$.

REMARK 6.19. If $\sigma_{1}, \ldots, \sigma_{\ell}$ are the facets of $\Delta$, then $\Delta=\mathcal{P}\left(\sigma_{1}\right) \cup \cdots \cup$ $\mathcal{P}\left(\sigma_{\ell}\right)$. In particular, a simplicial complex is determined by its facets.

Notation 6.20 . If $\Delta$ is a simplicial complex on the vertex set $[n]$ and $\sigma \in \Delta$, then $x^{\sigma}$ denotes $\prod_{i \in \sigma} x_{i} \in K\left[x_{1}, \ldots, x_{n}\right]$.

Definition 6.21 (Stanley-Reisner ideal of a simplicial complex). The Stanley-Reisner ideal of the simplicial complex $\Delta$ is the square-free monomial ideal $I_{\Delta}=\left(x^{\sigma} \mid \sigma \notin \Delta\right)$ of $K\left[x_{1}, \ldots, x_{n}\right]$. The Stanley-Reisner ring of $\Delta$ is $K\left[x_{1}, \ldots, x_{n}\right] / I_{\Delta}$.

Theorem 6.22 ([MS, Theorem 1.7]). The correspondence $\Delta \mapsto I_{\Delta}$ defines a bijection from simplicial complexes on the vertex set $[n]$ to square-free monomial ideals of $K\left[x_{1}, \ldots, x_{n}\right]$. Furthermore, $I_{\Delta}=\bigcap_{\sigma \in \Delta}\left(x^{[n] \backslash \sigma}\right)$. 
Proposition 6.23. Under the correspondence in Theorem 6.22, $I_{\Delta_{1} \cap \Delta_{2}}=$ $I_{\Delta_{1}}+I_{\Delta_{2}}$ and $I_{\Delta_{1} \cup \Delta_{2}}=I_{\Delta_{1}} \cap I_{\Delta_{2}}$ for all simplicial complexes $\Delta_{1}$ and $\Delta_{2}$.

Proof. For the first statement, we see that

$$
\begin{aligned}
x^{\sigma} \in I_{\Delta_{1} \cap \Delta_{2}} & \Leftrightarrow \sigma \notin \Delta_{1} \cap \Delta_{2} \Leftrightarrow \sigma \notin \Delta_{1} \text { or } \sigma \notin \Delta_{2} \\
& \Leftrightarrow x^{\sigma} \in I_{\Delta_{1}} \text { or } x^{\sigma} \in I_{\Delta_{1}} \Leftrightarrow x^{\sigma} \in I_{\Delta_{1}}+I_{\Delta_{2}} .
\end{aligned}
$$

The proof of the second statement is analogous.

TheOREm 6.24. Take a simplicial complex $\Delta$ on the vertex set $[n]$. Let $R$ be the Stanley-Reisner ring of $\Delta$, and let $m$ be its homogeneous maximal ideal. Then

$$
\chi_{\lambda}\left(R_{m}\right)=\sum_{i=-1}^{n}(-2)^{i+1}\left|F_{i}(\Delta)\right| .
$$

Proof. Let $S=K\left[x_{1}, \ldots, x_{n}\right]$, and let $\eta$ be its maximal homogeneous ideal. We proceed by induction on $d:=\operatorname{dim}(\Delta)$. If $d=0$, then $\Delta=\{\varnothing\}$. Then $I_{\Delta}=\eta$ and $R=K$, so that $\chi_{\lambda}\left(R_{m}\right)=1=(-2)^{0}=\sum_{i=-1}^{n}(-2)^{i+1}\left|F_{i}(\Delta)\right|$.

Assume that the formula holds for all simplicial complexes of dimension less than or equal to $d$. Take a simplicial complex $\Delta$ of dimension $d+1$. Consider all its facets, $\sigma_{1}, \ldots, \sigma_{\ell}$. We now proceed by induction on $\ell$. If $\ell=1$, suppose that $\Delta_{1}=\mathcal{P}\left(\sigma_{1}\right)$, where $\sigma_{1}=\left\{i_{1}, \ldots, i_{j}\right\}$ and $\operatorname{dim}\left(\sigma_{1}\right)=j$. Then $I_{\Delta_{1}}=\left(x_{i} \mid i \notin \sigma_{1}\right) S, R \cong K\left[x_{1}, \ldots, x_{n-j}\right]$, and

$$
\begin{aligned}
\chi_{\lambda}\left(R_{m}\right) & =\operatorname{length}_{D\left(\widehat{S}_{\eta}, K\right)} H_{I_{\Delta_{1}}}^{j}\left(\widehat{S}_{\eta}\right)=(-1)^{j}=(1-2)^{j}=\sum_{k=0}^{j} 1^{j-k}(-2)^{k}\left(\begin{array}{l}
j \\
k
\end{array}\right) \\
& =\sum_{k=-1}^{j-1}(-2)^{k+1}\left(\begin{array}{c}
j \\
k+1
\end{array}\right)=\sum_{k=-1}^{j-1}(-2)^{k+1}\left|F_{k}(\Delta)\right| .
\end{aligned}
$$

Assume that the formula is true for simplicial complexes of dimension $d+1$ with $\ell$ facets, and take a simplicial complex $\Delta$ of dimension $d+1$ with $\ell+1$ facets, $\sigma_{1}, \ldots, \sigma_{\ell}$. Let $\Delta_{i}=\mathcal{P}\left(\sigma_{i}\right)$, and let $\Delta^{\prime}=\Delta_{1} \cup \cdots \cup \Delta_{\ell}$. Then $\Delta=\Delta^{\prime} \cup \Delta_{\ell+1}$. We may assume, by renumbering, that $\operatorname{dim}\left(\Delta_{\ell}\right)=\operatorname{dim}(\Delta)$. Then $\operatorname{dim}\left(\Delta^{\prime} \cap \Delta_{\ell}\right)<\operatorname{dim}\left(\Delta_{\ell}\right)$ by our choice of $\Delta_{\ell}$ and as we chose the decomposition given by the facets. Therefore, $\chi_{\lambda}\left(R_{m}\right)$ equals

$$
\begin{aligned}
& \chi_{\lambda}\left(\left(S / I_{\Delta^{\prime} \cup \Delta_{\ell}}\right)_{\eta}\right) \\
& \quad=\chi_{\lambda}\left(\left(S / I_{\Delta^{\prime}} \cap I_{\Delta_{\ell}}\right)_{\eta}\right) \quad \text { by Proposition } 6.23
\end{aligned}
$$




$$
\begin{aligned}
= & \chi_{\lambda}\left(\left(S / I_{\Delta^{\prime}}\right)_{\eta}\right)+\chi_{\lambda}\left(\left(S / I_{\Delta_{\ell}}\right)_{\eta}\right) \\
& -\chi_{\lambda}\left(\left(S /\left(I_{\Delta^{\prime}}+I_{\Delta_{\ell}}\right)\right)_{\eta}\right) \quad \text { by Proposition } 4.16 \\
= & \chi_{\lambda}\left(\left(S / I_{\Delta^{\prime}}\right)_{\eta}\right)+\chi_{\lambda}\left(\left(S / I_{\Delta_{\ell}}\right)_{\eta}\right) \\
& -\chi_{\lambda}\left(\left(S /\left(I_{\Delta^{\prime} \cap \Delta_{\ell}}\right)\right)_{\eta}\right) \quad \text { by Proposition } 6.23 \\
= & \sum_{i=-1}^{n}(-2)^{i+1}\left|F_{i}\left(\Delta^{\prime}\right)\right|+\sum_{i=-1}^{n}(-2)^{i+1}\left|F_{i}\left(\Delta_{\ell}\right)\right| \\
& -\sum_{i=-1}^{n}(-2)^{i+1}\left|F_{i}\left(\Delta^{\prime} \cap \Delta_{\ell}\right)\right| \\
= & \sum_{i=-1}^{n}(-2)^{i+1}\left(\left|F_{i}\left(\Delta^{\prime}\right)\right|+\left|F_{i}\left(\Delta_{\ell}\right)\right|-\left|F_{i}\left(\Delta^{\prime} \cap \Delta_{\ell}\right)\right|\right) \\
= & \sum_{i=-1}^{n}(-2)^{i+1}\left|F_{i}\left(\Delta^{\prime} \cup \Delta_{\ell}\right)\right|=\sum_{i=-1}^{n}(-2)^{i+1}\left|F_{i}(\Delta)\right| .
\end{aligned}
$$

We note that [AGZ, Corollary 2.2], using [Mu, Theorem 3.3], shows that when $K$ has characteristic zero and when $R=K\left[x_{1}, \ldots, x_{n}\right]$ and $m=$ $\left(x_{1}, \ldots, x_{n}\right)$, then the $D(R, K)$-module characteristic cycle multiplicities of the local cohomology modules $H_{I_{\Delta}}^{i}(R)$ are determined by (and, in fact, determine) the graded Betti numbers of the Alexander dual of $I_{\Delta}$. By Theorem $6.10, \lambda_{I_{\Delta}}^{i}\left(R_{m}\right)=e\left(H_{I_{\Delta}}^{n-i}(R)\right)$, which determines the Lyubeznik characteristic $\chi_{\lambda}\left(\stackrel{R}{m}_{m}\right)$.

ExAmple 6.25. Let $K$ be a field, and let $S=K\left[x_{1}, x_{2}, x_{3}, x_{4}, x_{5}\right]$. Consider the ideal

$$
I=\left(x_{1} x_{3}, x_{1} x_{4}, x_{2} x_{3}, x_{2} x_{4}, x_{2} x_{5}\right)=\left(x_{1}, x_{2}\right) \cap\left(x_{2}, x_{3}, x_{4}\right) \cap\left(x_{3}, x_{4}, x_{5}\right)
$$

of $S$, so that $R:=S / I$ is the Stanley-Reisner ring of the simplicial complex in Figure 2. If $m=\left(x_{1}, x_{2}, x_{3}, x_{4}, x_{5}\right) \subseteq S$, Theorem 6.24 implies that $\chi_{\lambda}\left(R_{m}\right)=1 \cdot 1+(-2) \cdot 5+4 \cdot 5+(-8) \cdot 1=3$. This calculation can be confirmed by applying Proposition 4.16 iteratively using the primary decomposition of $I$.

Remark 6.26. In characteristic zero, Àlvarez Montaner [A1, Proposition 6.2] has given formulas for $\left|F_{i}(\Delta)\right|$ in terms of the characteristic cycle multiplicities of $H_{I_{\Delta}}^{j}\left(K\left[x_{1}, \ldots, x_{n}\right]\right)$. 


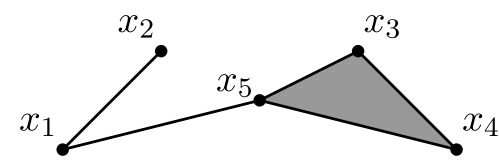

Figure 2: Simplicial complex in Example 6.25.

REMARK 6.27. Theorem 6.24 shows that the Lyubeznik characteristic of Stanley-Reisner rings does not depend on their characteristic, although their Lyubeznik numbers do have such a dependence (see [AV, Example 4.6]).

Acknowledgments. Many thanks go to Josep Àlvarez Montaner, Xavier Gómez-Mont, Daniel J. Hernández, Mel Hochster, and Felipe Pérez for useful mathematical conversations related to this work. We also thank Gennady Lyubeznik for pointing out an important observation regarding the definition of the new invariants. Finally, we thank the referees for useful comments and suggestions. Núñez-Betancourt's work was partially supported by National Council of Science and Technology of Mexico grant 210916. We are also grateful to the American Mathematical Society for supporting meetings between the authors through their Mathematical Research Communities program.

\section{REFERENCES}

[A1] J. Àlvarez Montaner, Characteristic cycles of local cohomology modules of monomial ideals, J. Pure Appl. Algebra 150 (2000), 1-25. MR 1762917. DOI 10.1016/S0022-4049(98)00171-6.

[A2] - Some numerical invariants of local rings, Proc. Amer. Math. Soc. 132 (2004), 981-986. MR 2045412. DOI 10.1090/S0002-9939-03-07177-6.

[AGZ] J. Àlvarez Montaner, R. García López, and S. Zarzuela Armengou, Local cohomology, arrangements of subspaces and monomial ideals, Adv. Math. 174 (2003), 35-56. MR 1959890. DOI 10.1016/S0001-8708(02)00050-6.

[AV] J. Àlvarez Montaner and A. Vahidi, Lyubeznik numbers of monomial ideals, Trans. Amer. Math. Soc. 366, no. 4 (2014), 1829-1855. MR 3152714. DOI 10.1090/S0002-9947-2013-05862-X.

[B1] J.-E. Björk, The global homological dimension of some algebras of differential operators, Invent. Math. 17 (1972), 67-78. MR 0320078.

[B2] , Rings of Differential Operators, North-Holland Math. Library 21, NorthHolland, Amsterdam, 1979. MR 0549189.

[Bl] M. Blickle, The intersection homology D-module in finite characteristic, Math. Ann. 328 (2004), 425-450. MR 2036330. DOI 10.1007/s00208-003-0492-z.

[BlB] M. Blickle and R. Bondu, Local cohomology multiplicities in terms of étale cohomology, Ann. Inst. Fourier (Grenoble) 55 (2005), 2239-2256. MR 2207383. 
[C] I. S. Cohen, On the structure and ideal theory of complete local rings, Trans. Amer. Math. Soc. 59 (1946), 54-106. MR 0016094.

[Co] S. C. Coutinho, A Primer of Algebraic D-Modules, London Math. Soc. Stud. Texts 33, Cambridge University Press, Cambridge, 1995. MR 1356713. DOI 10.1017/CBO9780511623653.

[GS] R. García López and C. Sabbah, Topological computation of local cohomology multiplicities, Collect. Math. 49 (1998), 317-324. MR 1677136.

[Gr] A. Grothendieck, Éléments de géométrie algébrique, IV: Étude locale des schémas et des morphismes de schémas, IV, Publ. Math. Inst. Hautes Études Sci. 32 (1967). MR 0238860.

[H] B. Haastert, On direct and inverse images of D-modules in prime characteristic, Manuscripta Math. 62 (1988), 341-354. MR 0966631. DOI 10.1007/BF01246838.

[Ha] R. Hartshorne, Local Cohomology, Lecture Notes in Math. 41, Springer, Berlin, 1967. MR 0224620.

[HH] M. Hochster and C. Huneke, Tight closure of parameter ideals and splitting in module-finite extensions. J. Algebraic Geom. 3 (1994), 599-670. MR 1297848.

[K1] K. Kawasaki, On the Lyubeznik number of local cohomology modules, Bull. Nara Univ. Ed. Natur. Sci. 49 (2000), 5-7. MR 1814657.

[K2] - On the highest Lyubeznik number, Math. Proc. Cambridge Philos. Soc. 132 (2002), 409-417. MR 1891679. DOI 10.1017/S0305004101005722.

[L1] G. Lyubeznik, Finiteness properties of local cohomology modules (an application of D-modules to commutative algebra), Invent. Math. 113 (1993), 41-55. MR 1223223. DOI 10.1007/BF01244301.

[L2] - Injective dimension of D-modules: A characteristic-free approach, J. Pure Appl. Algebra 149 (2000), 205-212. MR 1757731. DOI 10.1016/S0022-4049(98)00175-3.

[L3] - Finiteness properties of local cohomology modules: A characteristicfree approach, J. Pure Appl. Algebra 151 (2000), 43-50. MR 1770642. DOI 10.1016/S0022-4049(99)00080-8.

[L4] - "A partial survey of local cohomology" in Local Cohomology and Its Applications (Guanajuato, 1999), Lect. Notes Pure Appl. Math. 226, Dekker, New York, 2002, 121-154. MR 1888197.

[MN] Z. Mebkhout and L. Narváez-Macarro, La théorie du polynôme de Bernstein-Sato pour les algèbres de Tate et de Dwork-Monsky-Washnitzer, Ann. Sci. Éc. Norm. Supér. (4) 24 (1991), 227-256. MR 1097693.

[MS] E. Miller and B. Sturmfels, Combinatorial Commutative Algebra, Grad. Texts in Math. 227, Springer, New York, 2005. MR 2110098.

[Mu] M. Mustaţă, Local cohomology at monomial ideals, symbolic computation in algebra, analysis, and geometry (Berkeley, 1998), J. Symbolic Comput. 29 (2000), 709-720. MR 1769662. DOI 10.1006/jsco.1999.0302.

[NP] L. Núñez-Betancourt and F. Pérez, F-jumping and F-Jacobian ideals for hypersurfaces, preprint, arXiv:1302.3327v2 [math.AG]. DOI 10.1016/j.jpaa.2012.02.010.

[PS] C. Peskine and L. Szpiro, Dimension projective finie et cohomologie locale: Applications à la démonstration de conjectures de M. Auslander, H. Bass et A. Grothendieck, Publ. Math. Inst. Hautes Études Sci. (1973), 47-119. MR 0374130.

[V] J. C. Vassilev, Test ideals in quotients of F-finite regular local rings, Trans. Amer. Math. Soc. 350, no. 10 (1998), 4041-4051. MR 1458336. DOI 10.1090/S0002-9947-98-02128-X. 
[W1] U. Walther, On the Lyubeznik numbers of a local ring, Proc. Amer. Math. Soc. 129 (2001), 1631-1634. MR 1814090. DOI 10.1090/S0002-9939-00-05755-5.

[W2] , Bernstein-Sato polynomial versus cohomology of the Milnor fiber for generic hyperplane arrangements, Compos. Math. 141 (2005), 121-145. MR 2099772. DOI 10.1112/S0010437X04001149.

[Wi] E. E. Witt, Local cohomology with support in ideals of maximal minors, Adv. Math. 231 (2012), 1998-2012. MR 2964631. DOI 10.1016/j.aim.2012.07.001.

[Y1] K. Yanagawa, Alexander duality for Stanley-Reisner rings and squarefree $\mathbf{N}^{n}$-graded modules, J. Algebra 225 (2000), 630-645. MR 1741555. DOI 10.1006/jabr.1999.8130.

[Y2] - Bass numbers of local cohomology modules with supports in monomial ideals, Math. Proc. Cambridge Philos. Soc. 131 (2001), 45-60. MR 1833073. DOI 10.1017/S030500410100514X.

[Z] W. Zhang, On the highest Lyubeznik number of a local ring, Compos. Math. 143 (2007), 82-88. MR 2295196. DOI 10.1112/S0010437X06002387.

Luis Núñez-Betancourt

Department of Mathematics

University of Virginia

Charlottesville

Virginia 22904

USA

lcn8m@virginia.edu

Emily E. Witt

Department of Mathematics

University of Minnesota

Minneapolis

Minnesota 55455

$U S A$

ewitt@umn . edu 